\title{
Supramolecular Arene-Ruthenium Metallacycle with Thermotropic Liquid-Crystalline Properties
}

\author{
Cristina Alvariño Bouza, ${ }^{\dagger}$ Benoît Heinrich, ${ }^{\ddagger}$ Bertrand Donnio,,${ }^{*}{ }^{\ddagger}$ Robert Deschenaux, ${ }^{\dagger}$ and \\ Bruno Therrien ${ }^{*}+$
}

†Institut de Chimie, Université de Neuchâtel, Avenue de Bellevaux 51, 2000 Neuchâtel, Switzerland.

${ }^{\ddagger}$ Institut de Physique et Chimie des Matériaux de Strasbourg (IPCMS), UMR 7504, CNRS-Université de Strasbourg, 23 rue du Loess, BP43, 67034 Strasbourg cedex 2, France.

Table of Contents

Experimental section ............................................................................................................

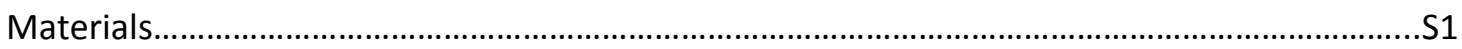

Techniques/Instrumentations................................................................................................

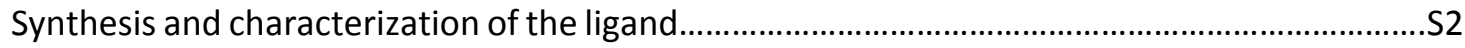

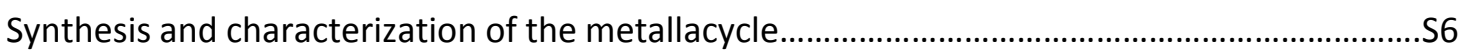

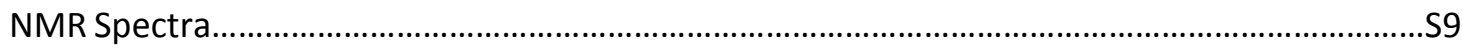

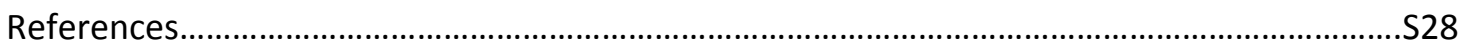

\section{Experimental section}

Materials. Compounds $1,{ }^{1} 4,{ }^{2} \mathbf{G}_{2}-\mathrm{OH}^{3}{ }^{3}$-(dimethylamino)pyridinium $p$-toluenesulfonate (DPTS), ${ }^{4}$ and $\left[\mathrm{Ru}_{2}(p \text {-cymene })_{2}(\text { donq })_{2} \mathrm{DDS}_{2}\right]^{5}$ were prepared according to published methods.

Techniques/Instrumentation. Column chromatography used silica gel 60 (63-200, $60 \AA$, Brunschwig). Mass spectra were obtained in positive-ion mode with a Bruker FTMS 4.7T BioAPEX II mass spectrometer by the spectrometry service at the University of Fribourg. The mass spectrum of the metallacycle was obtained by the group of Professor Schalley at the University of Berlin. Elemental analyzes were performed by the Mikroelementarisches Laboratorium, ETH Zürich. NMR measurements $\left({ }^{1} \mathrm{H},{ }^{13} \mathrm{C}, 2 \mathrm{D}\right.$ and DOSY) were recorded on a Bruker $\mathrm{AMX} 400$ spectrometer using the residual protonated solvent as internal standard. For DOSY experiments, the temperature was regulated at $21^{\circ} \mathrm{C}$, the airflow was increased to $670 \mathrm{I} \cdot \mathrm{min}^{-1}$, and the NMR tube was not spun. The diffusion NMR experiments were performed using a standard pulsed-gradient stimulated echo (LEDPFGSTE) sequence, using bipolar gradient.

Transition temperatures and enthalpies were determined with a differential scanning Mettler-Toledo DSC1 STARe System at a rate of $10^{\circ} \mathrm{C} \cdot \mathrm{min}^{-1}$ under $\mathrm{N}_{2}$. Optical studies were made using a Zeiss-Axioskop polarizing microscope equipped with a Linkam THMS-600 variable-temperature stage.

Laboratory SAXS patterns were recorded with a linear monochromatic Cu-K $\alpha 1$ beam $(\lambda=1.5405 \AA)$ obtained using a sealed-tube generator $(600 \mathrm{~W})$ equipped with a bent quartz monochromator. In all cases, the crude powder was filled in Lindemann capillaries of $1 \mathrm{~mm}$ diameter and $10 \mu \mathrm{m}$ wallthickness. The diffraction patterns were recorded with a curved Inel CPS120 counter gas-filled detector linked to a data acquisition computer (periodicities up to $90 \AA$ ) and on image plates scanned by 
Amersham Typhoon IP with $25 \mu \mathrm{m}$ resolution (periodicities up to $120 \AA$ ). The sample temperature was controlled within $\pm 0.01{ }^{\circ} \mathrm{C}$, and exposure times were varied from 4 to 6 hours.

\section{Synthesis and characterization of the ligand}

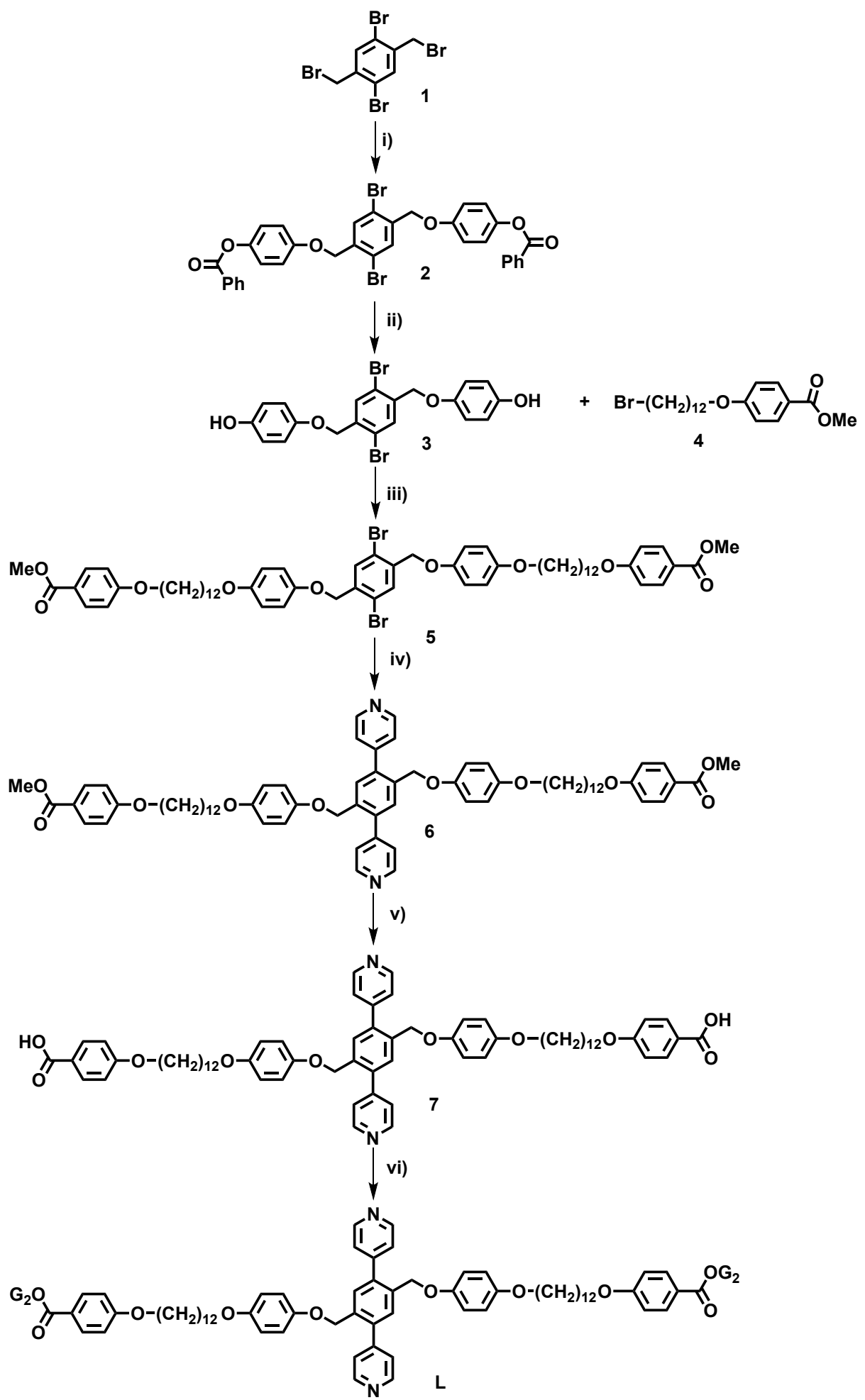

Scheme S1. Reagents and conditions: (i) 18-crown-6, 4-hydroxyphenyl benzoate, $\mathrm{K}_{2} \mathrm{CO}_{3}, \mathrm{CH}_{3} \mathrm{CN}$ (under reflux, overnight), yield: $42 \%$; (ii) $\mathrm{KOH}, \mathrm{THF} / \mathrm{H}_{2} \mathrm{O}$ (under reflux, overnight), yield: $95 \%$; (iii) 18 -crown-6, $\mathrm{K}_{2} \mathrm{CO}_{3}, \mathrm{CH}_{3} \mathrm{CN}$ (under reflux, overnight), yield: 73\%; (iv) 4-pyridinylboronic acid, $\mathrm{K}_{2} \mathrm{CO}_{3}, 1,4$-dioxane (under reflux, 8h), yield: $62 \%$; (v) $\mathrm{KOH}, \mathrm{THF} / \mathrm{H}_{2} \mathrm{O}$ (under reflux, overnight), yield: $78 \%$; (vi) $\mathrm{HO}-\mathrm{G}_{2}$, DPTS, $\mathrm{DCC}, \mathrm{CH}_{2} \mathrm{Cl}_{2}$, r.t., 24h, yield: $42 \%$. 
Synthesis of compound 2. To a mixture of 4-hydroxyphenyl benzoate ( $1.02 \mathrm{~g}, 4.76 \mathrm{mmol}), \mathrm{K}_{2} \mathrm{CO}_{3}$ (658 $\mathrm{mg}, 4.76 \mathrm{mmol}), 18$-crown-6 (314 mg, $0.119 \mathrm{mmol}$ ) in $\mathrm{CH}_{3} \mathrm{CN}(50 \mathrm{~mL})$ stirred at room temperature for $15 \mathrm{~min}$, was added 1 (1.00 g, $2.37 \mathrm{mmol})$. The reaction mixture was heated under reflux overnight. The solvent was removed under reduced pressure and the solid obtained was poured into distilled water. The powder was collected by filtration, washed $\left(\mathrm{H}_{2} \mathrm{O}\right)$ and dried under vacuum. The crude product was purified by flash column chromatography (silica gel, cyclohexane $/ \mathrm{CH}_{2} \mathrm{Cl}_{2}: 4 / 6$ ) to obtain $685 \mathrm{mg}(42 \%)$ of 2 as a white powder. ${ }^{1} \mathrm{H}$ NMR $\left(400 \mathrm{MHz}, \mathrm{CD}_{2} \mathrm{Cl}_{2}\right) \delta 8.21(\mathrm{~d}, J=7.7 \mathrm{~Hz}, 4 \mathrm{H}), 7.88(\mathrm{~s}, 2 \mathrm{H}), 7.69(\mathrm{t}, J=7.6$ $\mathrm{Hz}, 2 \mathrm{H}), 7.56(\mathrm{t}, J=7.7 \mathrm{~Hz}, 4 \mathrm{H}), 7.21(\mathrm{~d}, J=7.6 \mathrm{~Hz}, 4 \mathrm{H}), 7.10(\mathrm{~d}, J=7.9 \mathrm{~Hz}, 4 \mathrm{H}), 5.17(\mathrm{~s}, 4 \mathrm{H}) .{ }^{13} \mathrm{C} \mathrm{NMR}$ $\left(101 \mathrm{MHz}, \mathrm{CD}_{2} \mathrm{Cl}_{2}\right) \delta 165.34,155.93,145.12,137.60,133.51,132.42,129.95,129.62,128.55,122.69$, 121.16, 115.50, 69.15. Anal. Calcd for $\mathrm{C}_{34} \mathrm{H}_{24} \mathrm{Br}_{2} \mathrm{O}_{6}$ : C, 59.32; H, 3.51. Found: $\mathrm{C}, 59.25 ; \mathrm{H}, 3.56$. ESI-MS: $\mathrm{m} / \mathrm{z}$ calcd for $[\mathrm{M}+\mathrm{Na}]^{+} 710.98$, found: 711.2 .

Synthesis of compound 3. Compound $2(0.48 \mathrm{~g}, 0.70 \mathrm{mmol})$ was hydrolyzed with $\mathrm{KOH}(2.45 \mathrm{~g}, 43.6$ $\mathrm{mmol})$ in a THF/ $\mathrm{H}_{2} \mathrm{O}(15 / 30 \mathrm{~mL})$ mixture. The latter was heated under reflux overnight. THF was removed under reduced pressure and $\mathrm{HCl}$ was added to $\mathrm{pH}=8$. The precipitate was filtered and washed $\left(\mathrm{H}_{2} \mathrm{O}\right)$. The white solid was dried under vacuum to obtain $321 \mathrm{mg}(95 \%)$ of 3. ${ }^{1} \mathrm{H} \mathrm{NMR}\left(400 \mathrm{MHz}, \mathrm{CDCl}_{3}\right.$ and a drop of DMSO) $\delta 6.67(\mathrm{~s}, 2 \mathrm{H}), 7.26(\mathrm{~d}, J=8.9 \mathrm{~Hz}, 4 \mathrm{H}), 6.76-6.69(\mathrm{~d}, J=\mathrm{m}, 8 \mathrm{H}), 5.02(\mathrm{~s}, 2 \mathrm{H}), 4.53$ $(\mathrm{s}, 4 \mathrm{H}) .{ }^{13} \mathrm{C}$ NMR $\left(101 \mathrm{MHz}, \mathrm{CDCl}_{3}\right.$ and a drop of DMSO) $\delta 151.79,151.34,137.81,132.22,120.89$, 116.14, 115.97, 69.50. Anal. Calcd for $\mathrm{C}_{20} \mathrm{H}_{16} \mathrm{Br}_{2} \mathrm{O}_{4}$ : C, 50.03; H, 3.36. Found: $\mathrm{C}, 50.23 ; \mathrm{H}, 3.57$. ESI-MS: $\mathrm{m} / \mathrm{z}$ calcd for [M-H]- 478.93, found: 479.3 .

Synthesis of compound 5. To a mixture of 3 ( $400 \mathrm{mg}, 0.833 \mathrm{mmol}), \mathrm{K}_{2} \mathrm{CO}_{3}(230 \mathrm{mg}, 1.67 \mathrm{mmol}), 18-$ crown-6 (11.0 mg, $0.0416 \mathrm{mmol})$ in $\mathrm{CH}_{3} \mathrm{CN}(16 \mathrm{~mL}$ ) stirred at room temperature for $15 \mathrm{~min}$, was added $4(0.667 \mathrm{~g}, 1.67 \mathrm{mmol})$. The reaction mixture was heated under reflux overnight. The solvent was removed under reduced pressure and water was added. The solid was filtered and washed $\left(\mathrm{H}_{2} \mathrm{O}\right)$. The white solid was dried under vacuum to obtain $679 \mathrm{mg}(73 \%)$ of compound $\left.5 .{ }^{1} \mathrm{H} \mathrm{NMR} \mathrm{(400} \mathrm{MHz}, \mathrm{CDCl}_{3}\right)$ $7.98(\mathrm{~d}, J=8.3 \mathrm{~Hz}, 4 \mathrm{H}), 7.77(\mathrm{~s}, 2 \mathrm{H}), 7.00-6.78(\mathrm{~m}, 12 \mathrm{H}), 5.03(\mathrm{~s}, 4 \mathrm{H}), 4.00(\mathrm{t}, J=6.5 \mathrm{~Hz}, 4 \mathrm{H}), 3.91(\mathrm{t}, J$ $=7.0 \mathrm{~Hz}, 4 \mathrm{H}), 3.88(\mathrm{~s}, 6 \mathrm{H}), 1.78(\mathrm{~m}, J=14.3,6.9 \mathrm{~Hz}, 8 \mathrm{H}), 1.50-1.39(\mathrm{~m}, 8 \mathrm{H}), 1.39-1.23(\mathrm{~m}, 24 \mathrm{H}) .{ }^{13} \mathrm{C}$ NMR $\left(101 \mathrm{MHz}, \mathrm{CDCl}_{3}\right) \delta 166.91,162.97,153.96,152.25,137.78,132.29,131.56,122.36,120.98$, $115.93,115.52,114.08,69.48,68.65,68.23,51.81,29.56,29.41,29.40,29.36,29.14,26.07,26.00$. Anal. Calcd for $\mathrm{C}_{60} \mathrm{H}_{76} \mathrm{O}_{10} \mathrm{Br}_{2}: \mathrm{C}, 64.51 ; \mathrm{H}, 6.86$. Found: $\mathrm{C}, 64.28 ; \mathrm{H}, 6.79$. ESI-MS: $\mathrm{m} / \mathrm{z}$ calcd for $[\mathrm{M}+\mathrm{Na}]^{+}$ 1139.4, found: 1139.5 .

Synthesis of compound 6. A mixture of 4-pyridinylboronic acid (116 mg, $0.940 \mathrm{mmol}), \mathrm{K}_{2} \mathrm{CO}_{3}(130 \mathrm{mg}$, $0.940 \mathrm{mmol}), 5(300 \mathrm{mg}, 0.269 \mathrm{mmol})$, and $\mathrm{Pd}\left(\mathrm{PPh}_{3}\right)_{4}(0.0622 \mathrm{mg}, 0.0538 \mathrm{mmol})$ in 1,4-dioxane (3.5 $\mathrm{mL}$ ) was stirred at $100^{\circ} \mathrm{C}$ for $8 \mathrm{~h}$ under $\mathrm{N}_{2}$. After removal of the solvent by reduced pressure, the solid was purified by flash column chromatography (silica gel, $\mathrm{CH}_{2} \mathrm{Cl}_{2} / \mathrm{AcOEt}$ : 9:1) to obtain $187 \mathrm{mg}$ (62\%) of 6. ${ }^{1} \mathrm{H}$ NMR $\left(400 \mathrm{MHz}, \mathrm{CD}_{2} \mathrm{Cl}_{2}\right) \delta 8.66(\mathrm{~d}, J=5.1 \mathrm{~Hz}, 4 \mathrm{H}), 7.97(\mathrm{~d}, J=8.6 \mathrm{~Hz}, 4 \mathrm{H}), 7.64(\mathrm{~s}, 2 \mathrm{H}), 7.41(\mathrm{~d}, J$ $=5.3 \mathrm{~Hz}, 4 \mathrm{H}), 6.93(\mathrm{~d}, J=8.5 \mathrm{~Hz}, 4 \mathrm{H}), 6.80(\mathrm{~s}, 8 \mathrm{H}), 4.94(\mathrm{~s}, 4 \mathrm{H}), 4.03(\mathrm{t}, J=6.6 \mathrm{~Hz}, 4 \mathrm{H}), 3.89(\mathrm{t}, J=6.8 \mathrm{~Hz}$, $4 \mathrm{H}), 3.87(\mathrm{~s}, 6 \mathrm{H}), 1.84-1.81(\mathrm{~m}, 8 \mathrm{H}), 1.46(\mathrm{~s}, 8 \mathrm{H}), 1.32(\mathrm{~s}, 24 \mathrm{H}) .{ }^{13} \mathrm{C} \mathrm{NMR}\left(101 \mathrm{MHz}, \mathrm{CD}_{2} \mathrm{Cl}_{2}\right) \delta 166.58$, $162.97,153.83,152.15,149.79,147.43,139.50,134.56,131.33,131.06,122.32,115.73,115.19$, $114.00,68.50,68.26,68.19,51.62,29.54,29.52,29.38,29.33,29.32,29.08$. Anal. Calcd for $\mathrm{C}_{70} \mathrm{H}_{84} \mathrm{~N}_{2} \mathrm{O}_{10}$ : C, $75.51 ; H, 7.60 ; N, 2.52$. Found: $C, 75.45 ; H, 7.51 ; N, 2.53$. ESI-MS: $m / z$ calcd for $[M+H]^{+} 1113.6$, found: 1113.8 .

Synthesis of compound 7. Compound $6(150 \mathrm{mg}, 0.135 \mathrm{mmol})$ and $\mathrm{KOH}(468 \mathrm{mg}, 8.35 \mathrm{mmol})$ in a $\mathrm{THF} / \mathrm{H}_{2} \mathrm{O}(3 / 6 \mathrm{~mL})$ mixture was heated under reflux overnight. THF was removed under reduced pressure and $\mathrm{HCl}$ was added to neutral $\mathrm{pH}$. The precipitate was filtered and washed $\left(\mathrm{H}_{2} \mathrm{O}\right)$. The white solid was dried under vacuum to obtain $114 \mathrm{mg}(78 \%)$ of 7. ${ }^{1} \mathrm{H} \mathrm{NMR} \mathrm{(400} \mathrm{MHz,} \mathrm{CDCl}_{3}$ and a drop of DMSO) $\delta 8.58(\mathrm{~d}, J=4.5 \mathrm{~Hz}, 4 \mathrm{H}), 7.89(\mathrm{~d}, J=8.4 \mathrm{~Hz}, 4 \mathrm{H}), 7.52(\mathrm{~s}, 2 \mathrm{H}), 7.31(\mathrm{~s}, 4 \mathrm{H}), 6.81(\mathrm{~d}, J=8.3 \mathrm{~Hz}$, 
$4 \mathrm{H}), 6.70(\mathrm{~s}, 8 \mathrm{H}), 4.82(\mathrm{~s}, 4 \mathrm{H}), 3.92(\mathrm{t}, J=6.5 \mathrm{~Hz}, 4 \mathrm{H}), 3.80(\mathrm{t}, J=6.5 \mathrm{~Hz}, 4 \mathrm{H}), 3.61(\mathrm{~s}, 2 \mathrm{H}), 1.68(\mathrm{dt}, J=$ $18.5,7.4 \mathrm{~Hz}, 8 \mathrm{H}), 1.35(\mathrm{~d}, J=7.6 \mathrm{~Hz}, 8 \mathrm{H}), 1.20(\mathrm{~s}, 24 \mathrm{H}) .{ }^{13} \mathrm{C} \mathrm{NMR}\left(100 \mathrm{MHz}, \mathrm{CDCl}_{3}\right.$ and a drop of DMSO) $\delta 168.28,162.71,153.79,152.04,149.37,147.87,139.42,134.48,131.68,131.25,124.10,122.91$, $115.78,115.35,113.89,68.51,68.22,68.08,29.60,29.44,29.26,29.02,25.95,25.88$. Anal. Calcd for $\mathrm{C}_{68} \mathrm{H}_{80} \mathrm{~N}_{2} \mathrm{O}_{10}: \mathrm{C}, 75.25 ; \mathrm{H}, 7.43 ; \mathrm{N}, 2.58$. Found: $\mathrm{C}, 75.45 ; \mathrm{H}, 7.54 ; \mathrm{N}, 2.43$. ESI-MS: $\mathrm{m} / \mathrm{z}$ calcd for $[\mathrm{M}+\mathrm{H}]^{+}$: 1085.59, found: 1085.5 .

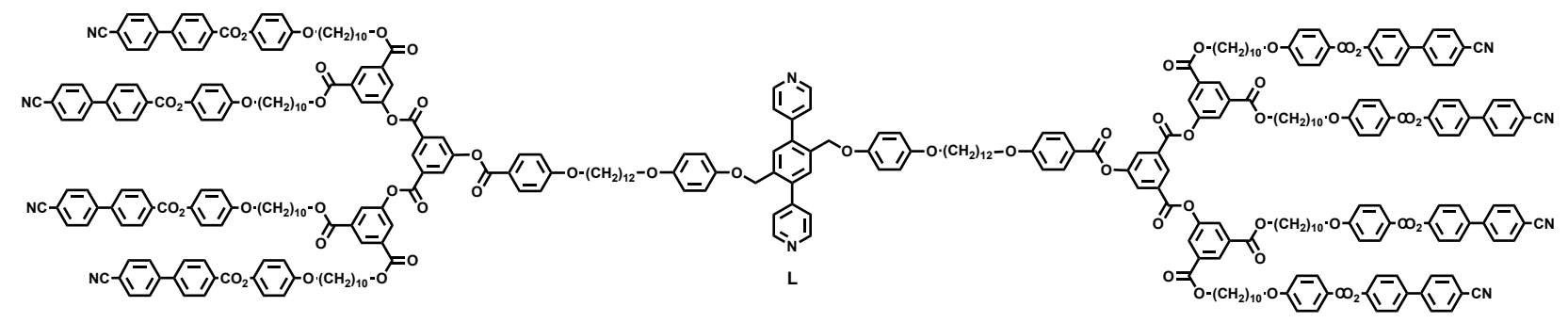

Synthesis of L. To a mixture of $\mathbf{G}_{2}-\mathrm{OH}\left(470 \mathrm{mg}, 0.202 \mathrm{mmol}\right.$ ) and $\mathbf{7}\left(100 \mathrm{mg}, 0.0918 \mathrm{mmol}\right.$ ) in dry $\mathrm{CH}_{2} \mathrm{Cl}_{2}$ $\left(10 \mathrm{~mL}\right.$ ) cooled to $0{ }^{\circ} \mathrm{C}$, was added $N, N^{\prime}$-dicyclohexylcarbodiimide (DCC) $(56.9 \mathrm{mg}, 0.276 \mathrm{mmol})$ and 4(dimethylamino)pyridinium toluene-p-sulfonate (DPTS) $(40.6 \mathrm{mg}, 0.138 \mathrm{mmol})$. After stirring at room temperature for 24 hours, the solvent was removed and the residue was purified by column chromatography (silica gel, $\mathrm{CH}_{2} \mathrm{Cl}_{2} / \mathrm{AcOEt} 100 / 0.5$ to 100/2.5). The solvent was evaporated under reduced pressure and the isolated product dried under vacuum to obtain $220 \mathrm{mg}(42 \%)$ of $\mathrm{L}$. ${ }^{1} \mathrm{H}$ NMR $\left(400 \mathrm{MHz}, \mathrm{CD}_{2} \mathrm{Cl}_{2}\right) \delta 8.93(\mathrm{~s}, 2 \mathrm{H}), 8.64(\mathrm{~d}, J=5.2 \mathrm{~Hz}, 4 \mathrm{H}), 8.60(\mathrm{~s}, 4 \mathrm{H}), 8.36(\mathrm{~s}, 4 \mathrm{H}), 8.17(\mathrm{~d}, J=9.2 \mathrm{~Hz}$, $4 \mathrm{H}), 8.15-8.03(\mathrm{~m}, 24 \mathrm{H}), 7.80-7.69(\mathrm{~m}, 32 \mathrm{H}), 7.66(\mathrm{~d}, J=8.2 \mathrm{~Hz}, 16 \mathrm{H}), 7.61(\mathrm{~s}, 2 \mathrm{H}), 7.39(\mathrm{~d}, J=5.1 \mathrm{~Hz}$, $4 \mathrm{H}), 7.32(\mathrm{~d}, J=8.2 \mathrm{~Hz}, 16 \mathrm{H}), 6.98(\mathrm{~d}, J=8.6 \mathrm{~Hz}, 20 \mathrm{H}), 6.78(\mathrm{~s}, 8 \mathrm{H}), 4.92(\mathrm{~s}, 4 \mathrm{H}), 4.36(\mathrm{t}, J=6.7 \mathrm{~Hz}, 16 \mathrm{H})$, $4.04(\mathrm{t}, J=6.4 \mathrm{~Hz}, 2 \mathrm{H}), 3.87(\mathrm{t}, J=6.7 \mathrm{~Hz}, 4 \mathrm{H}), 1.94-1.63(\mathrm{~m}, 40 \mathrm{H}), 1.56-1.42(\mathrm{~m}, 40 \mathrm{H}), 1.33(\mathrm{~d}, J=$ $19.3 \mathrm{~Hz}, 88 \mathrm{H}) .{ }^{13} \mathrm{C} N M R\left(101 \mathrm{MHz}, \mathrm{CD}_{2} \mathrm{Cl}_{2}\right) \delta 164.72,164.64,164.32,164.12,163.72,163.12,153.88$, $152.21,151.73,151.66,150.66,149.71,147.54,144.62,139.49,136.62,134.59,132.71,132.62$, $132.37,132.14,131.17,131.06,128.88,128.73,128.23,128.04,127.60,126.79,124.01,122.51$, $121.24,120.41,118.75,115.78,115.25,114.50,114.32,111.00,68.54,68.42,68.25,65.83,29.67$, 29.57, 29.43, 29.41, 29.28, 29.20, 29.06, 28.60, 26.02, 25.94, 25.91. Anal. Calcd for $\mathrm{C}_{356} \mathrm{H}_{352} \mathrm{~N}_{10} \mathrm{O}_{58}$ : C, 75.03; H, 6.23; N, 2.46. Found: $\mathrm{C}, 74.74 ; \mathrm{H}, 6.44 ; \mathrm{N}, 2.51$. MALDI-TOF-MS: $\mathrm{m} / \mathrm{z}$ calcd for $[\mathrm{M}+\mathrm{Na}]^{+}$ 5721.49, found: 5721.12 . 


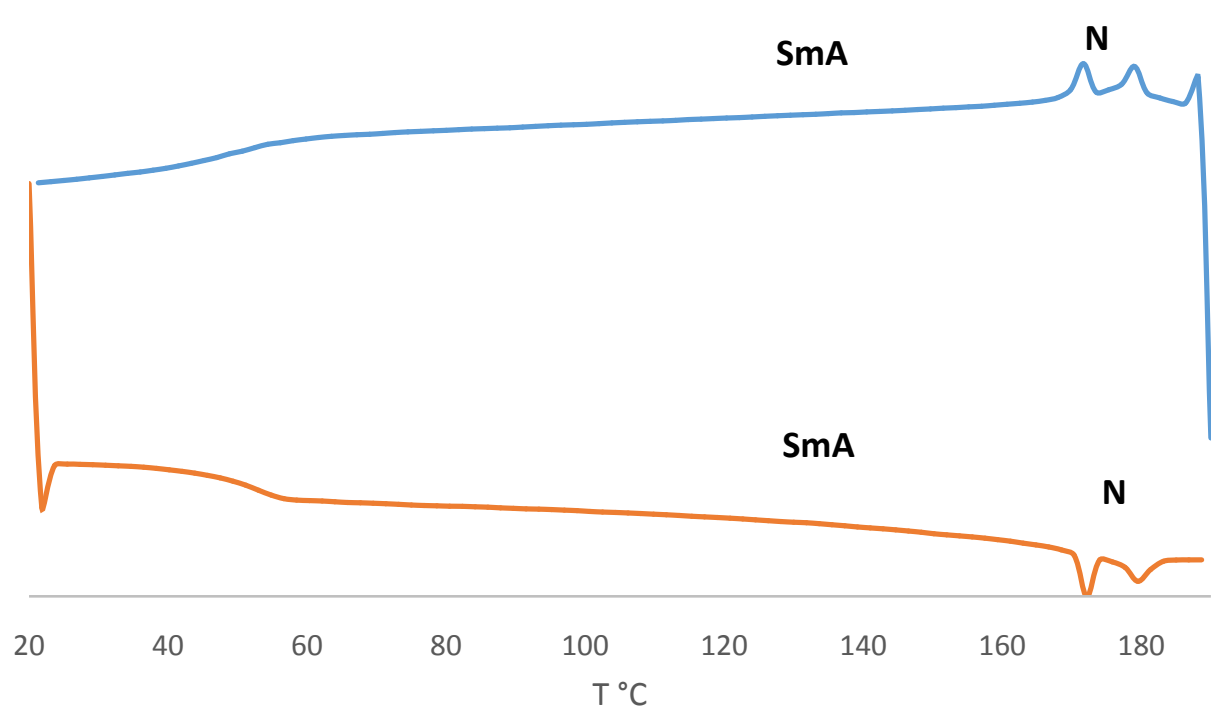

Figure S1. Differential scanning thermograms of compound $\mathbf{L}$ registered during the second heating (bottom) cooling (top) cycle.

\begin{tabular}{|c|c|c|c|c|}
\hline Compound & $\mathrm{T}_{\mathrm{g}}{ }^{a}$ & Transition $^{a}$ & Temperature $\left({ }^{\circ} \mathrm{C}\right)$ & $\Delta \mathrm{H}\left[\mathrm{kJ} \cdot \mathrm{mol}^{-1}\right]$ \\
\hline $\mathbf{L}$ & 56 & $\mathrm{SmA} \rightarrow \mathrm{N}$ & 172 & 3.75 \\
& 5 & $\mathrm{~N} \rightarrow \mathrm{I}$ & 180 & 5.84 \\
\hline
\end{tabular}

Table S1. Phase transition temperatures of $\mathbf{L} .{ }^{a} \mathrm{~T}_{\mathrm{g}}$ : glass transition temperature. Temperatures are given as the onset of the peaks obtained during the second heating run; $\mathrm{T}_{\mathrm{g}}$ was determined during the first cooling cycle (rate: $\left.10^{\circ} \mathrm{C} \cdot \mathrm{min}^{-1}\right)$. 
Synthesis and characterization of the metallacycle

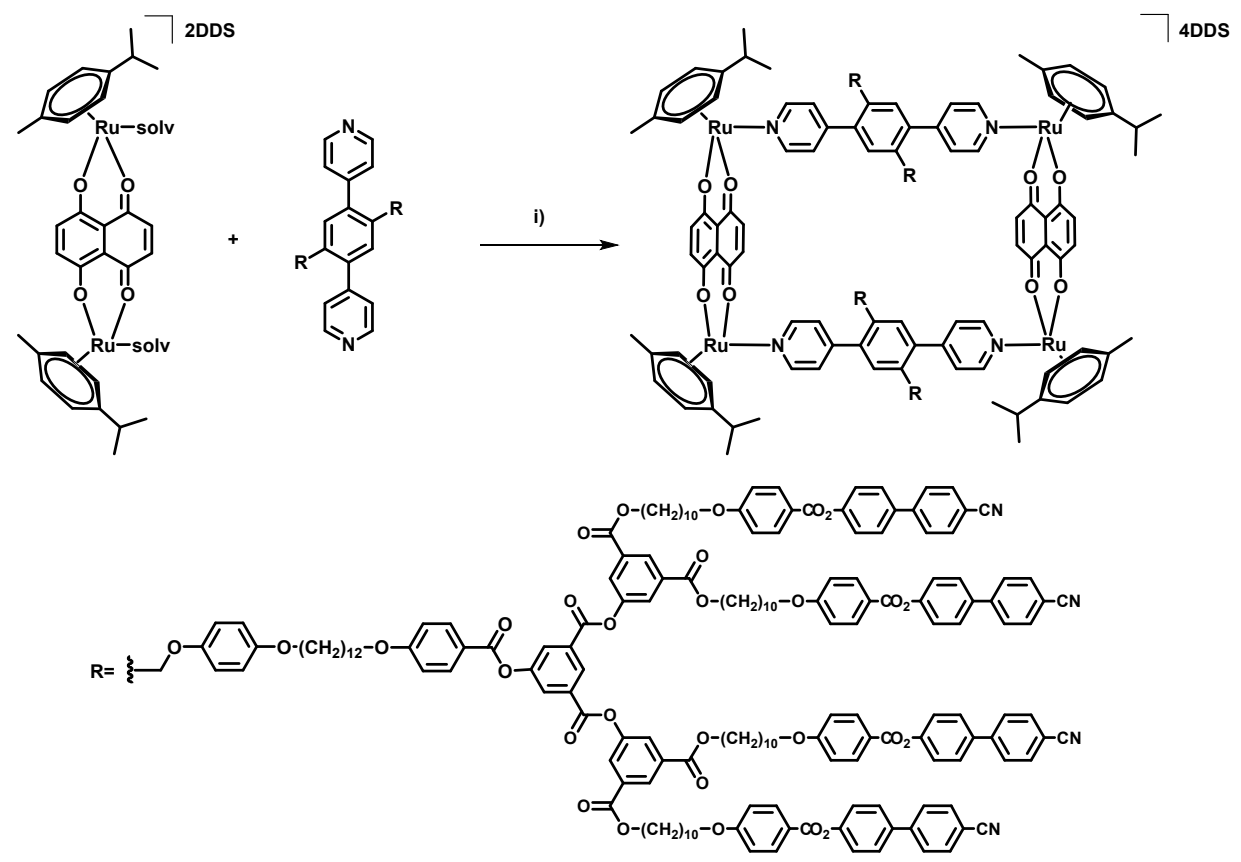

Scheme S2. Reagents and conditions: (i) $\mathrm{CH}_{2} \mathrm{Cl}_{2}$, r.t., $24 \mathrm{~h}$, yield: $93 \%$.

DDS: dodecylsulfate.

A mixture of $\left[\mathrm{Ru}_{2}(p \text {-cymene })_{2}(\mathrm{donq})_{2} \mathrm{DDS}_{2}\right](21.4 \mathrm{mg}, 0.0180 \mathrm{mmol})$ and $\mathbf{L}(103 \mathrm{mg}, 0.0180 \mathrm{mmol})$ in $\mathrm{CH}_{2} \mathrm{Cl}_{2}(2 \mathrm{~mL})$ was stirred at room temperature for 24 hours. Diethyl ether was added and the precipitate was isolated by filtration as a green dark solid. Yield: $93 \%(115 \mathrm{mg}) .{ }^{1} \mathrm{H} \mathrm{NMR}(400 \mathrm{MHz}$, $\left.\mathrm{CD}_{2} \mathrm{Cl}_{2}\right) \delta 8.93(\mathrm{~s}, 4 \mathrm{H}), 8.60(\mathrm{~s}, 8 \mathrm{H}), 8.40(\mathrm{~d}, J=5.0 \mathrm{~Hz}, 8 \mathrm{H}), 8.36(\mathrm{~s}, 8 \mathrm{H}), 8.17(\mathrm{~d}, J=8.8 \mathrm{~Hz}, 8 \mathrm{H}), 8.15-$ $8.06(\mathrm{~m}, 48 \mathrm{H}), 7.79-7.69(\mathrm{~m}, 64 \mathrm{H}), 7.66(\mathrm{~d}, J=8.2 \mathrm{~Hz}, 32 \mathrm{H}), 7.32(\mathrm{~d}, J=8.3 \mathrm{~Hz}, 44 \mathrm{H}), 7.29-7.17(\mathrm{~m}$, $8 \mathrm{H}), 6.98(\mathrm{~d}, J=8.3 \mathrm{~Hz}, 40 \mathrm{H}), 6.52(\mathrm{t}, J=8.2 \mathrm{~Hz}, 8 \mathrm{H}), 6.39(\mathrm{t}, J=9.5 \mathrm{~Hz}, 8 \mathrm{H}), 5.68(\mathrm{~m}, 8 \mathrm{H}), 5.55(\mathrm{~m}, 8 \mathrm{H})$, $4.72(\mathrm{br}, 8 \mathrm{H}), 4.36(\mathrm{t}, J=6.7 \mathrm{~Hz}, 32 \mathrm{H}), 4.04(\mathrm{t}, J=6.4 \mathrm{~Hz}, 40 \mathrm{H}), 3.88(\mathrm{~m}, 16 \mathrm{H}), 2.84-2.64(\mathrm{~m}, 4 \mathrm{H}), 2.08$ $(\mathrm{s}, 6 \mathrm{H}), 2.07(\mathrm{~s}, 6 \mathrm{H}), 1.80(\mathrm{~m}, 80 \mathrm{H}), 1.60(\mathrm{t}, J=6.9 \mathrm{~Hz}, 8 \mathrm{H}), 1.48(\mathrm{~m}, 80 \mathrm{H}), 1.36(\mathrm{~s}, 176 \mathrm{H}), 1.26(\mathrm{~m}, 96 \mathrm{H})$, $0.88(\mathrm{t}, J=6.5 \mathrm{~Hz}, 12 \mathrm{H}) .{ }^{13} \mathrm{C} \mathrm{NMR}\left(101 \mathrm{MHz}, \mathrm{CD}_{2} \mathrm{Cl}_{2}\right) \delta 170.96,164.72,164.64,164.31,164.11,163.72$, $163.11,153.77,153.70,151.73,151.66,151.60,151.46,150.65,150.49,144.62,138.16,138.11$, $137.53,137.40,136.60,135.02,134.94,132.70,132.62,132.38,132.14,131.84,131.60,131.17$, $128.87,128.72,128.23,128.03,127.60,126.79,126.35,122.52,121.23,120.42,118.76,115.62$, $115.40,115.10,115.07,114.48,114.32,111.68,111.64,110.98,103.69,103.67,99.57,99.46,84.32$, $84.16,82.93,68.60,68.56,68.42,66.99,65.83,31.92,30.70,29.76,29.70,29.66,29.58,29.52,29.50$, 29.44, 29.4, 29.36, 29.29, 29.20, 29.06, 28.60, 26.20, 26.03, 25.94, 25.91, 22.68, 21.96, 17.06, 13.90. Anal. Calcd for $\mathrm{C}_{820} \mathrm{H}_{868} \mathrm{~N}_{20} \mathrm{O}_{140} \mathrm{Ru}_{4} \mathrm{~S}_{4}$ : C, 71.49; H, 6.35; N, 2.03. Found: C, 71.20; H, 6.52; N, 2.02. ESIMS: $\mathrm{m} / \mathrm{z}$ calcd for [M-4DDS] ${ }^{+4} 3178.77$, found: 3178.68 . 


\begin{tabular}{|c|c|c|c|c|c|c|c|}
\hline & $T^{\circ} \mathrm{C}$ & Phase & $\begin{array}{c}V_{\text {mol }}\left[10^{3} \AA^{3}\right] \\
\left(\rho\left[\mathrm{g} / \mathrm{cm}^{3}\right]\right)\end{array}$ & $d(\AA)$ & $A_{\text {mol }}\left(\AA^{2}\right)$ & $a_{\text {mes }}\left(\AA^{2}\right)$ & $\tau$ \\
\hline $\mathbf{L}$ & 20 & SmA & $8.19(1.15)$ & $54.4 \pm 0.2$ & 151 & 37.7 & 0.29 \\
$\mathbf{L}$ & 60 & $\mathrm{SmA}$ & $8.44(1.12)$ & $53.9 \pm 0.2$ & 156 & 39.1 & 0.27 \\
$\mathbf{L}$ & 80 & $\mathrm{SmA}$ & $8.56(1.10)$ & $53.0 \pm 0.2$ & 161 & 40.4 & 0.24 \\
$\mathbf{L}$ & 100 & $\mathrm{SmA}$ & $8.68(1.09)$ & $52.1 \pm 0.2$ & 167 & 41.7 & 0.21 \\
$\mathbf{M}_{\mathbf{2}} \mathbf{L}_{\mathbf{2}}$ & 20 & Lam & $19.66(1.16)$ & $95.4 \pm 0.4$ & 208 & 26.0 & 0.82 \\
$\mathbf{M}_{\mathbf{2}} \mathbf{L}_{\mathbf{2}}$ & 60 & Lam & $20.25(1.13)$ & $94.9 \pm 0.4$ & 213 & 26.7 & 0.82 \\
$\mathbf{M}_{\mathbf{2}} \mathbf{L}_{\mathbf{2}}$ & 80 & Lam & $20.54(1.11)$ & $95.0 \pm 0.4$ & 216 & 27.0 & 0.82 \\
$\mathbf{M}_{\mathbf{2}} \mathbf{L}_{\mathbf{2}}$ & 100 & Lam & $20.84(1.10)$ & $95.3 \pm 0.4$ & 219 & 27.3 & 0.83 \\
\hline
\end{tabular}

Table S2: Structural parameters for the multilayered smectic A (SmA) phase of $\mathbf{L}$ and multilayered smectic-like lamellar phase of $\mathbf{M}_{\mathbf{2}} \mathbf{L}_{\mathbf{2}} . V_{\mathrm{mol}}$ is the molecular volume, $\rho$ the density, $d$ the lamellar spacing, $A_{\mathrm{mol}}$ the molecular area, $a_{\text {mes }}$ the area per mesogen, and $\tau=2-a_{\text {mes }} / \sigma_{\text {mes, }}$, the bilayering ratio of the mesogen layers (as defined in references ${ }^{6}$ ).
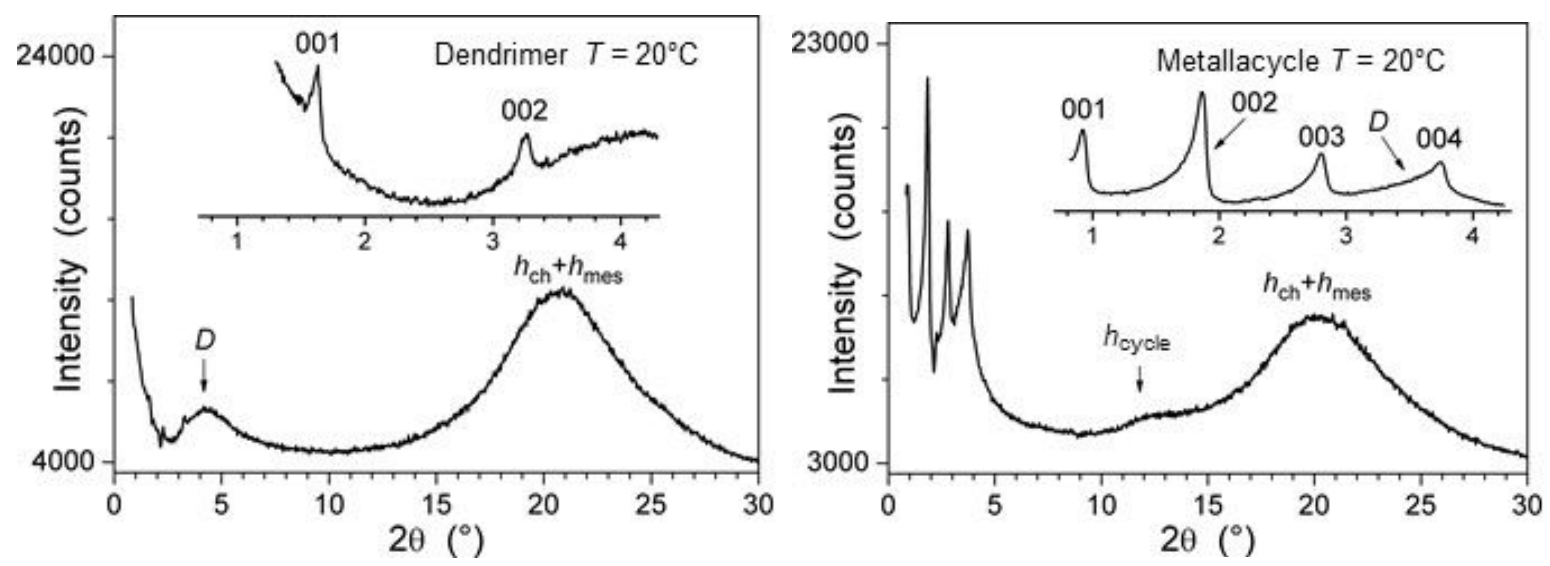

Figure S2. SAXS patterns of $\mathbf{L}$ (left) and $\mathbf{M}_{\mathbf{2}} \mathbf{L}_{\mathbf{2}}$ (right) in pristine state $\left(20^{\circ} \mathrm{C}\right.$ ). 

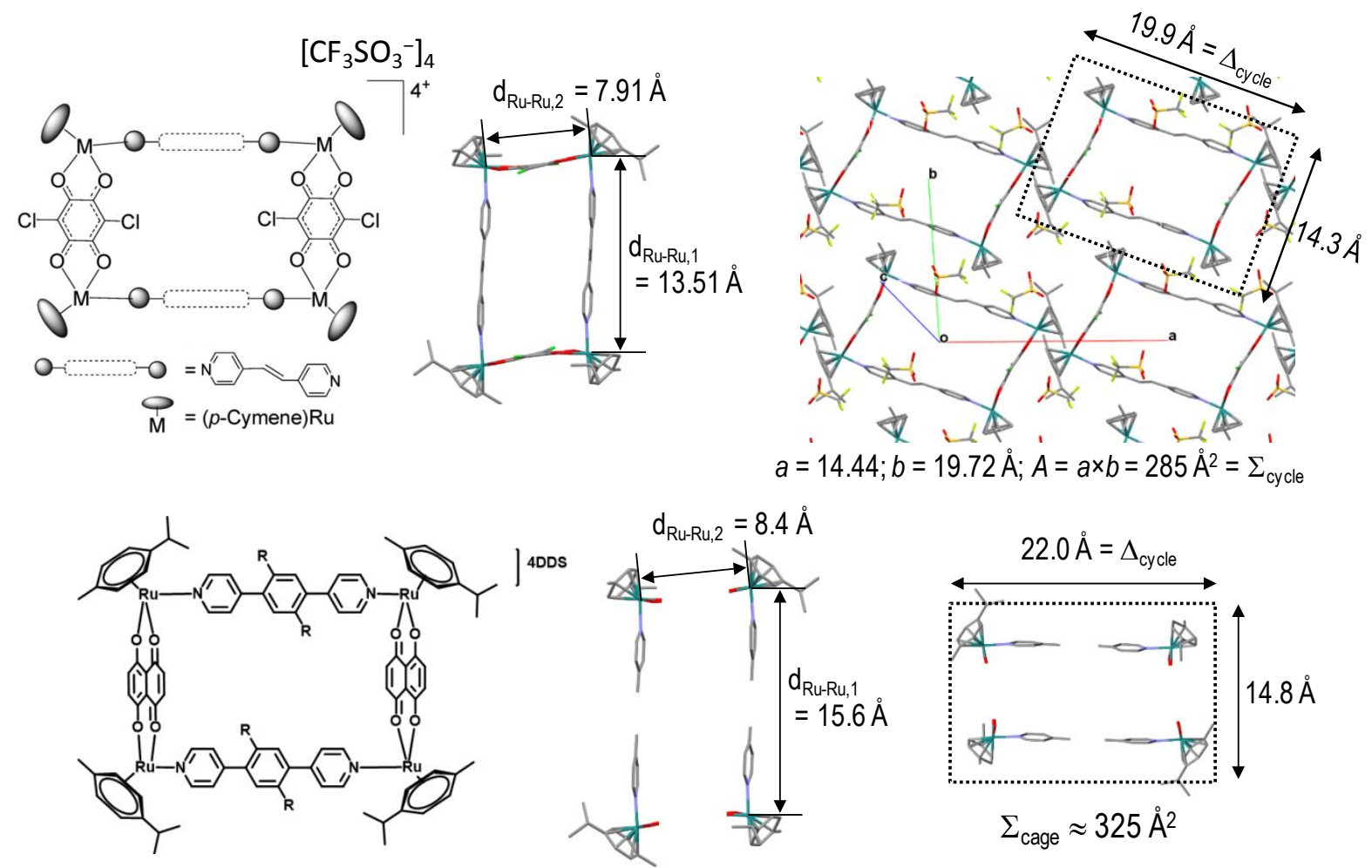

Figure S3. Top: molecular formula, molecular conformation and self-assembling of a model metallacycle in the reference single-crystal structure CSD-EGARAG; ${ }^{7}$ the arrangement in the $\mathbf{a} \times \mathbf{b}$ sub-lattice gives access to the crosssectional area of a molecule in the plane of the metallacycle, $\Sigma_{\text {cycle }}$, and to the average spacing of cycles in parallel to long sides, $\Delta_{\text {cycle }}$. Bottom: molecular formula of $\mathbf{M}_{\mathbf{2}} \mathbf{L}_{\mathbf{2}}$, which involves slightly longer metallacyclic ligands, resulting in somewhat larger metallacycle dimensions and estimated $\Sigma_{\text {cycle }}$ and $\Delta_{\text {cycle }}$ values. 
NMR Spectra
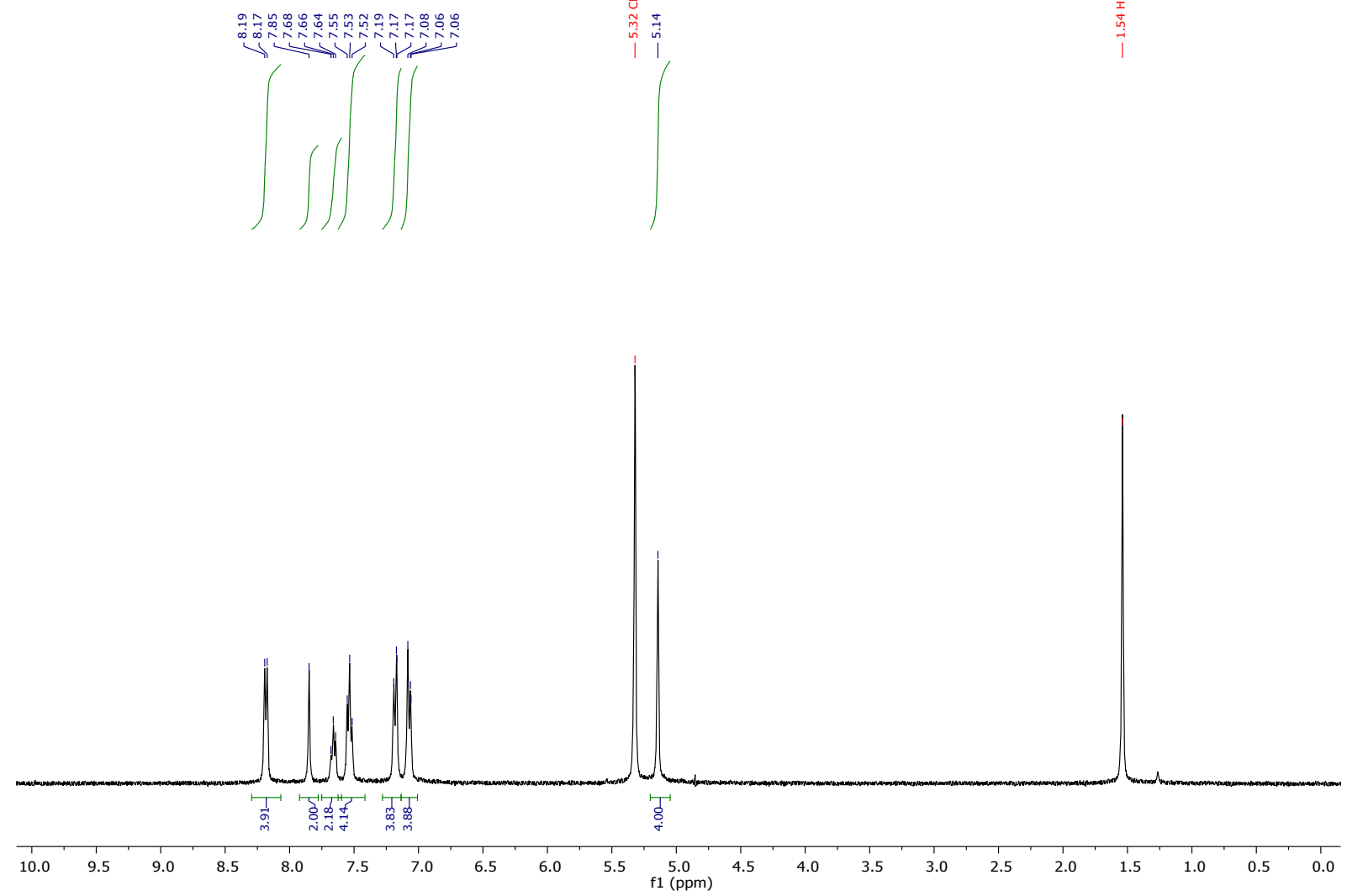

Figure S4. ${ }^{1} \mathrm{H}$ NMR spectrum of compound $2\left(400 \mathrm{MHz}, \mathrm{CD}_{2} \mathrm{Cl}_{2}, 298 \mathrm{~K}\right)$.
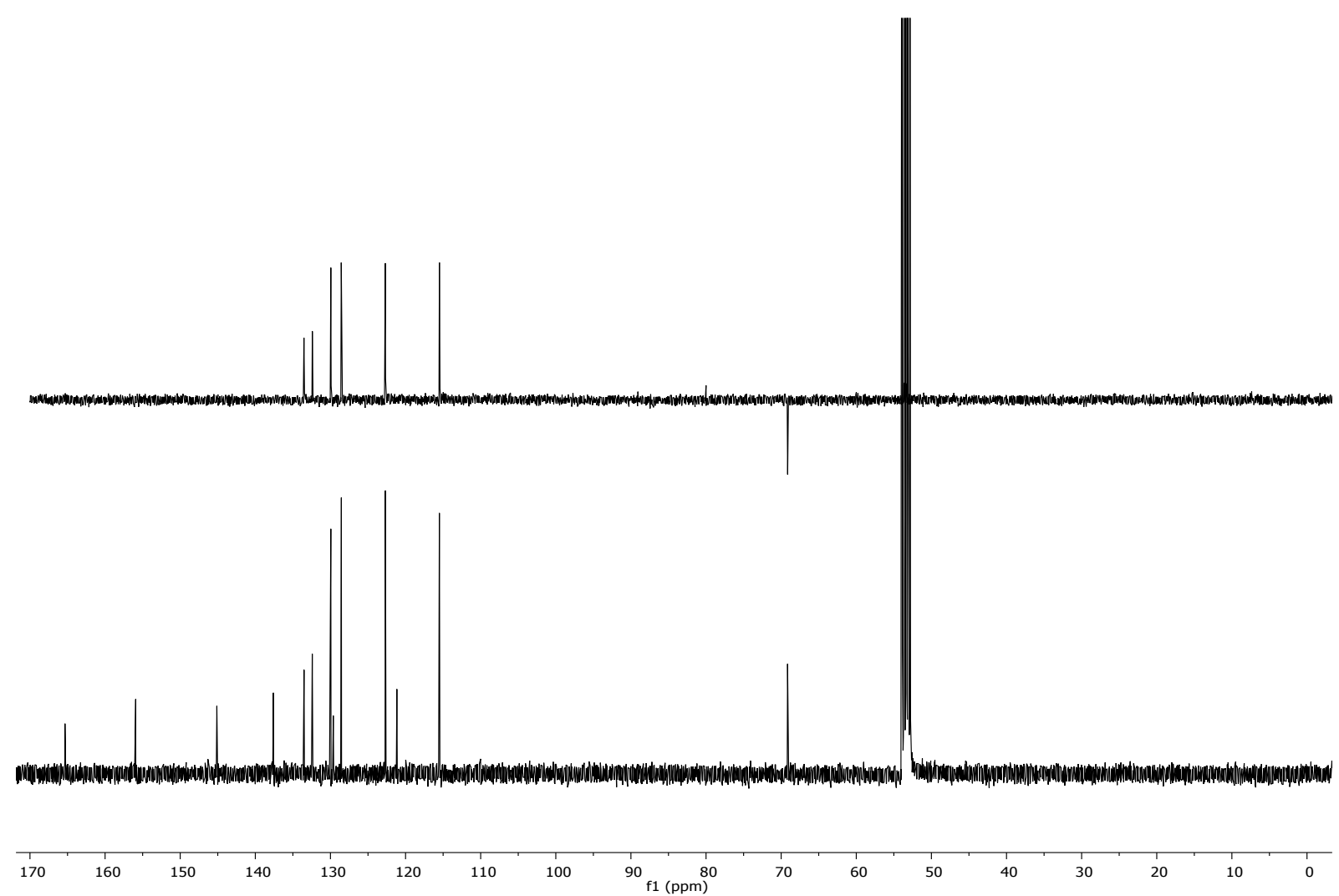

Figure S5. DEPT and ${ }^{13} \mathrm{C}$ NMR spectra of compound $2\left(100 \mathrm{MHz}, \mathrm{CD}_{2} \mathrm{Cl}_{2}, 298 \mathrm{~K}\right.$ ). 


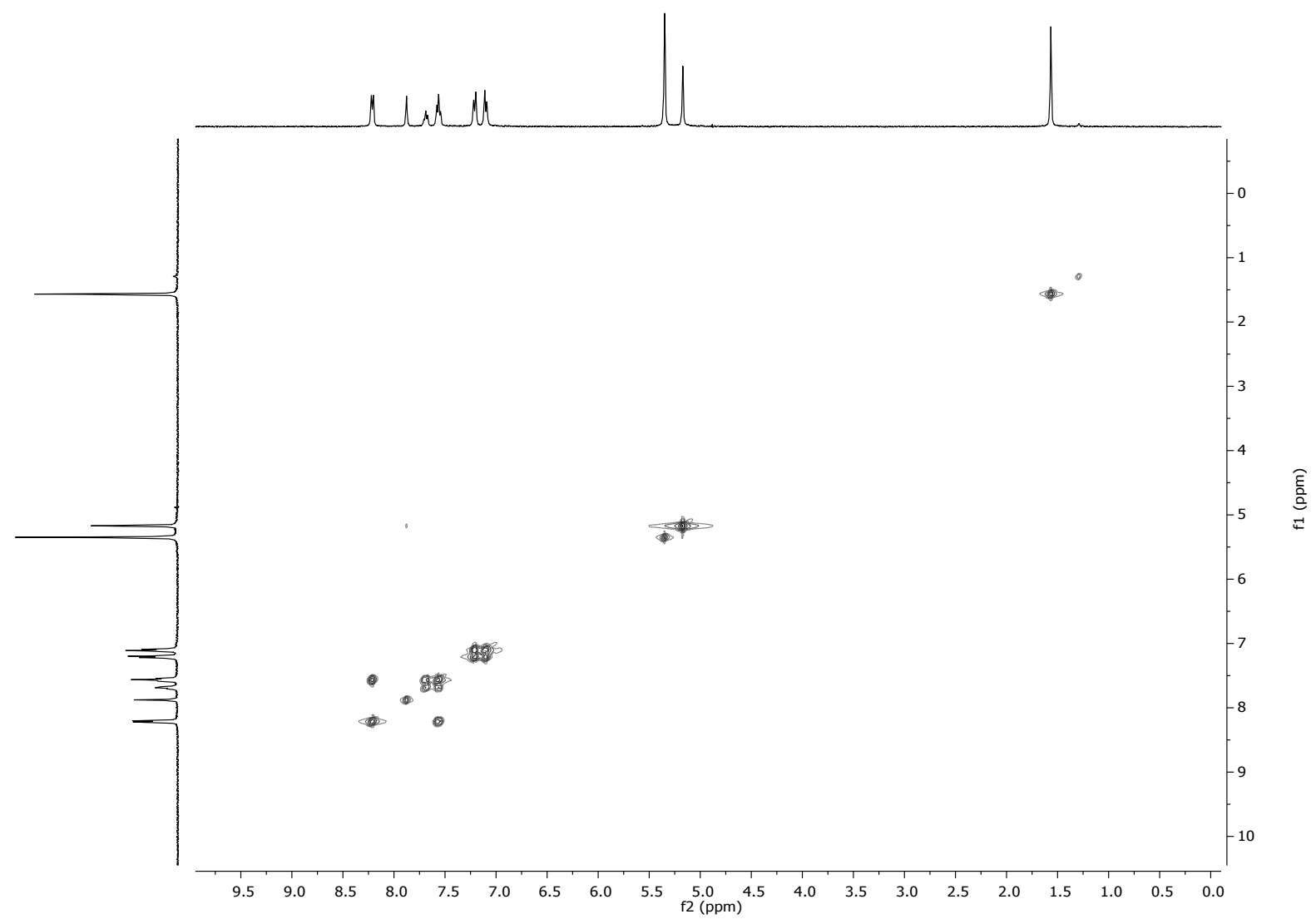

Figure S6. 2D COSY spectrum of compound 2 (400 MHz, $\mathrm{CD}_{2} \mathrm{Cl}_{2}, 298 \mathrm{~K}$ ).

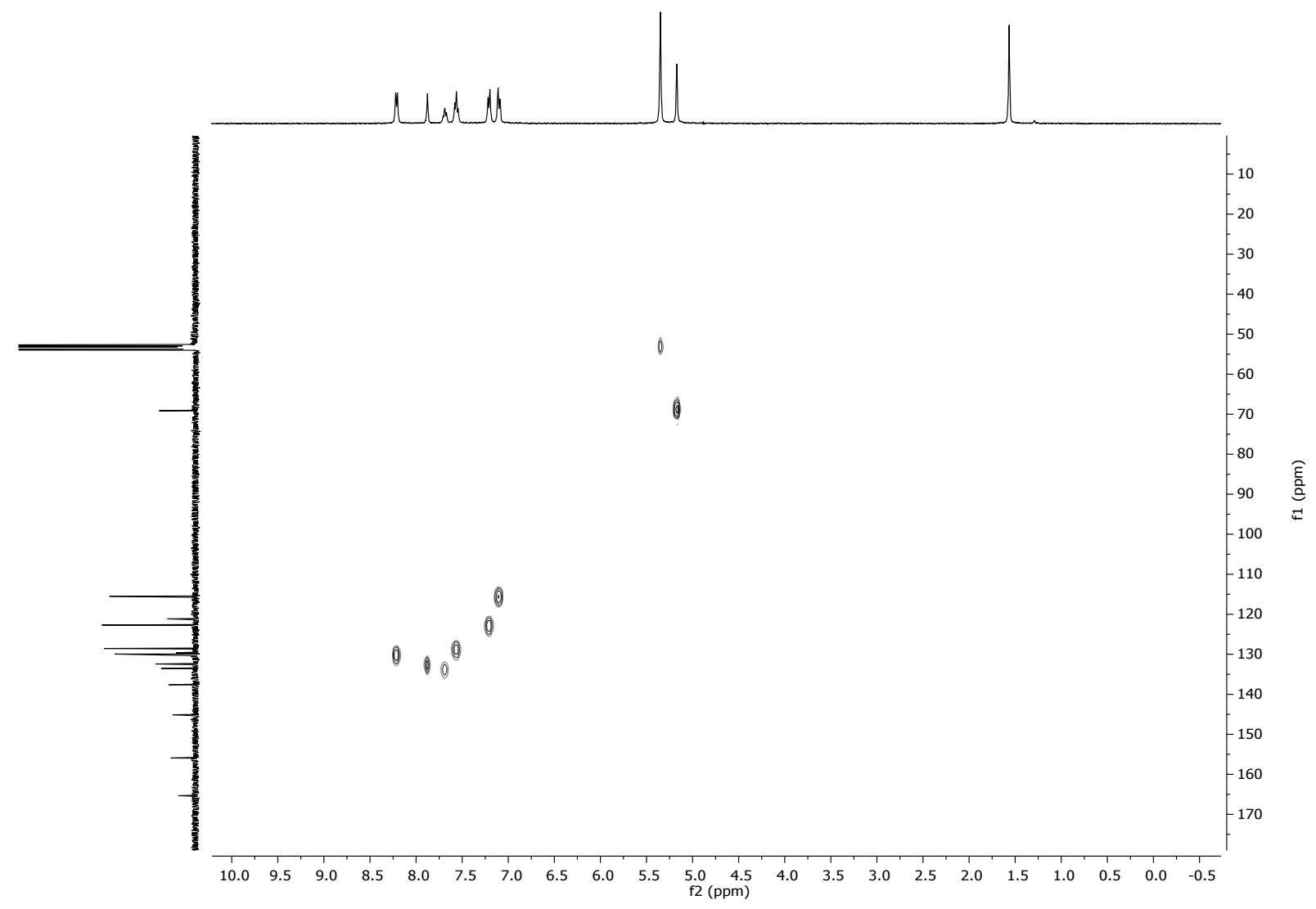

Figure S7. 2D HSQC spectrum of compound $2\left(400 \mathrm{MHz}, \mathrm{CD}_{2} \mathrm{Cl}_{2}, 298 \mathrm{~K}\right)$. 


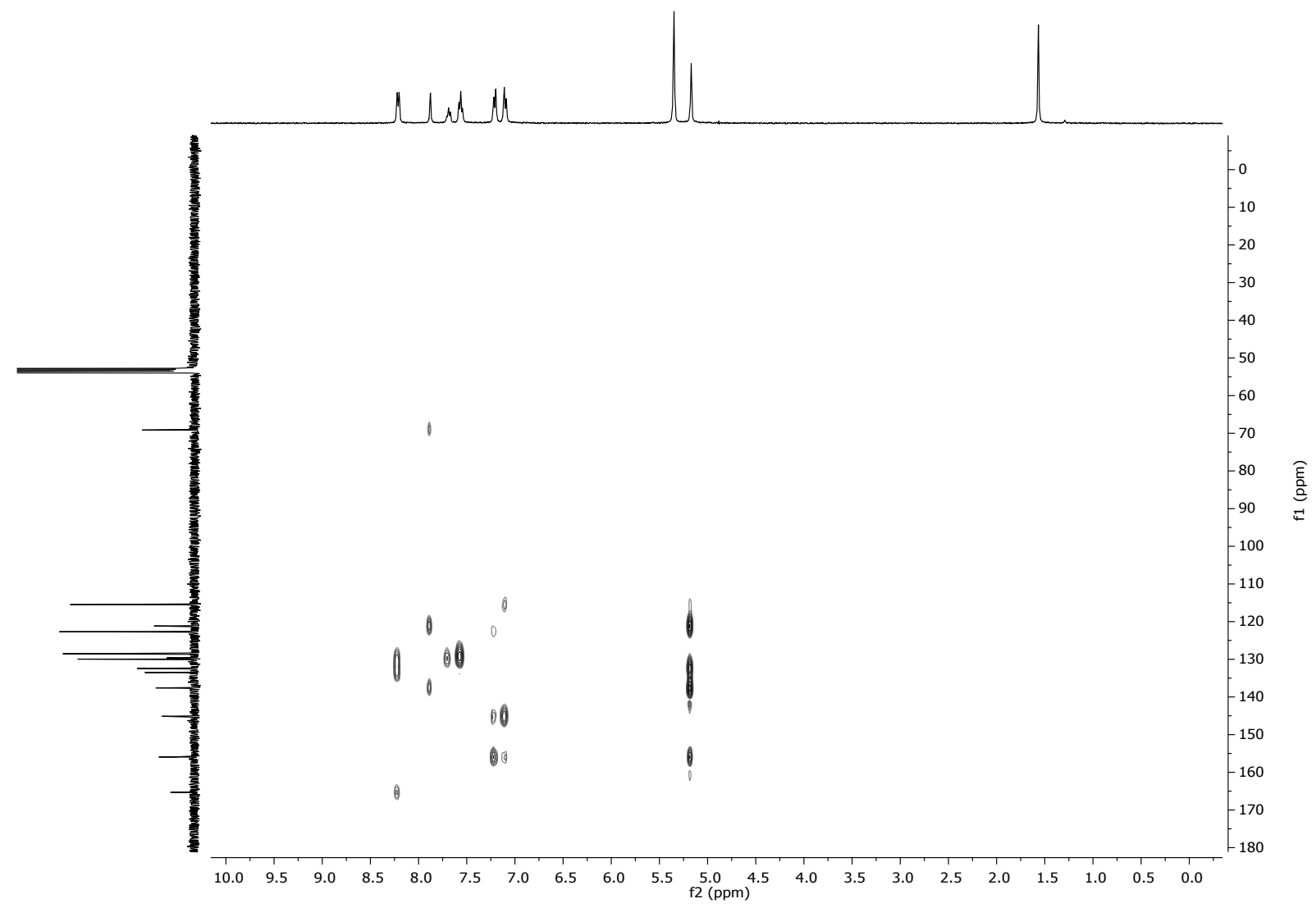

Figure S8. 2D HMBC spectrum of compound 2 (400 MHz, $\left.\mathrm{CD}_{2} \mathrm{Cl}_{2}, 298 \mathrm{~K}\right)$.

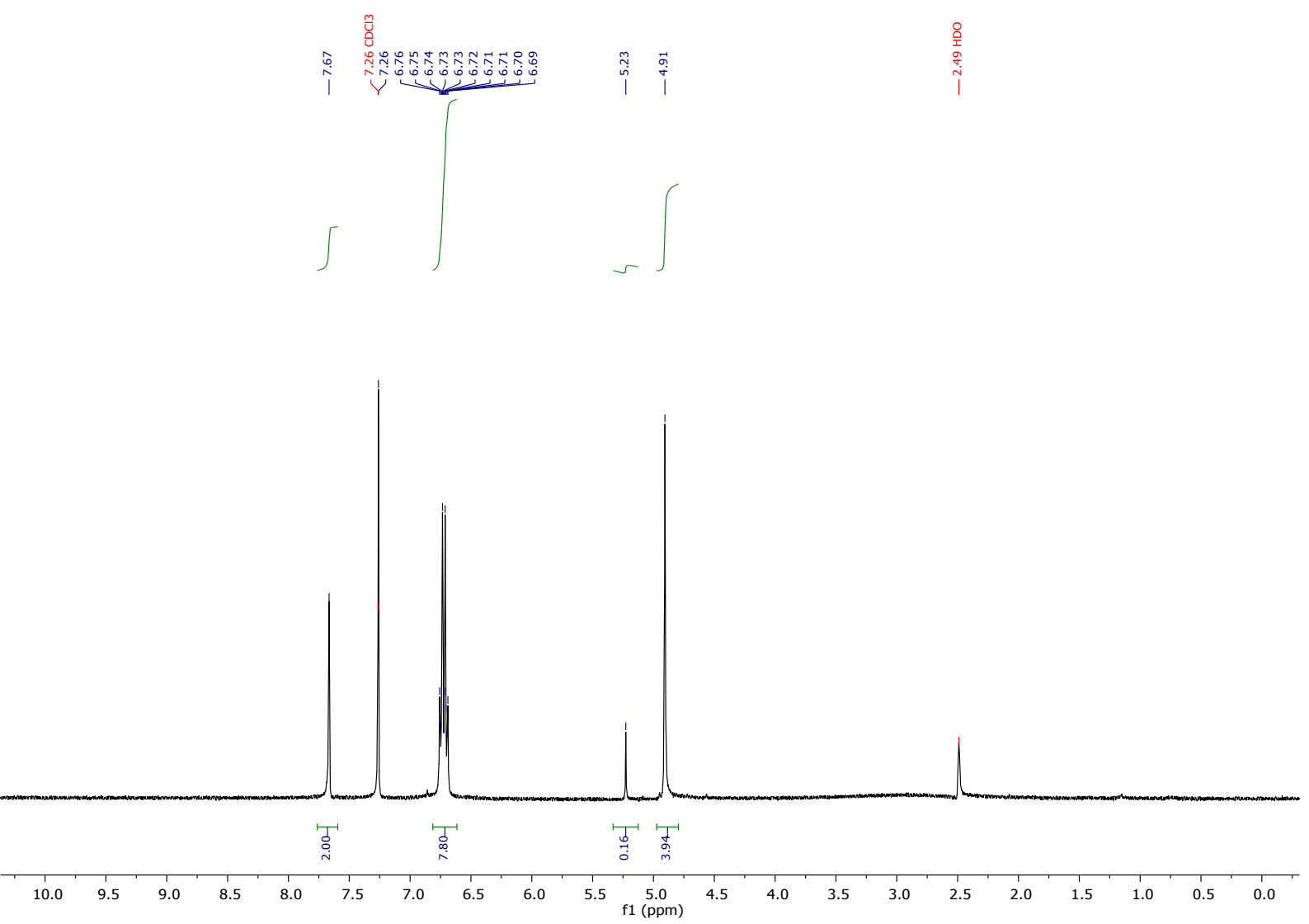

Figure S9. ${ }^{1} \mathrm{H}$ NMR spectrum of compound 3 (400 MHz, CDCl 3 and a drop of DMSO, $298 \mathrm{~K}$ ). 

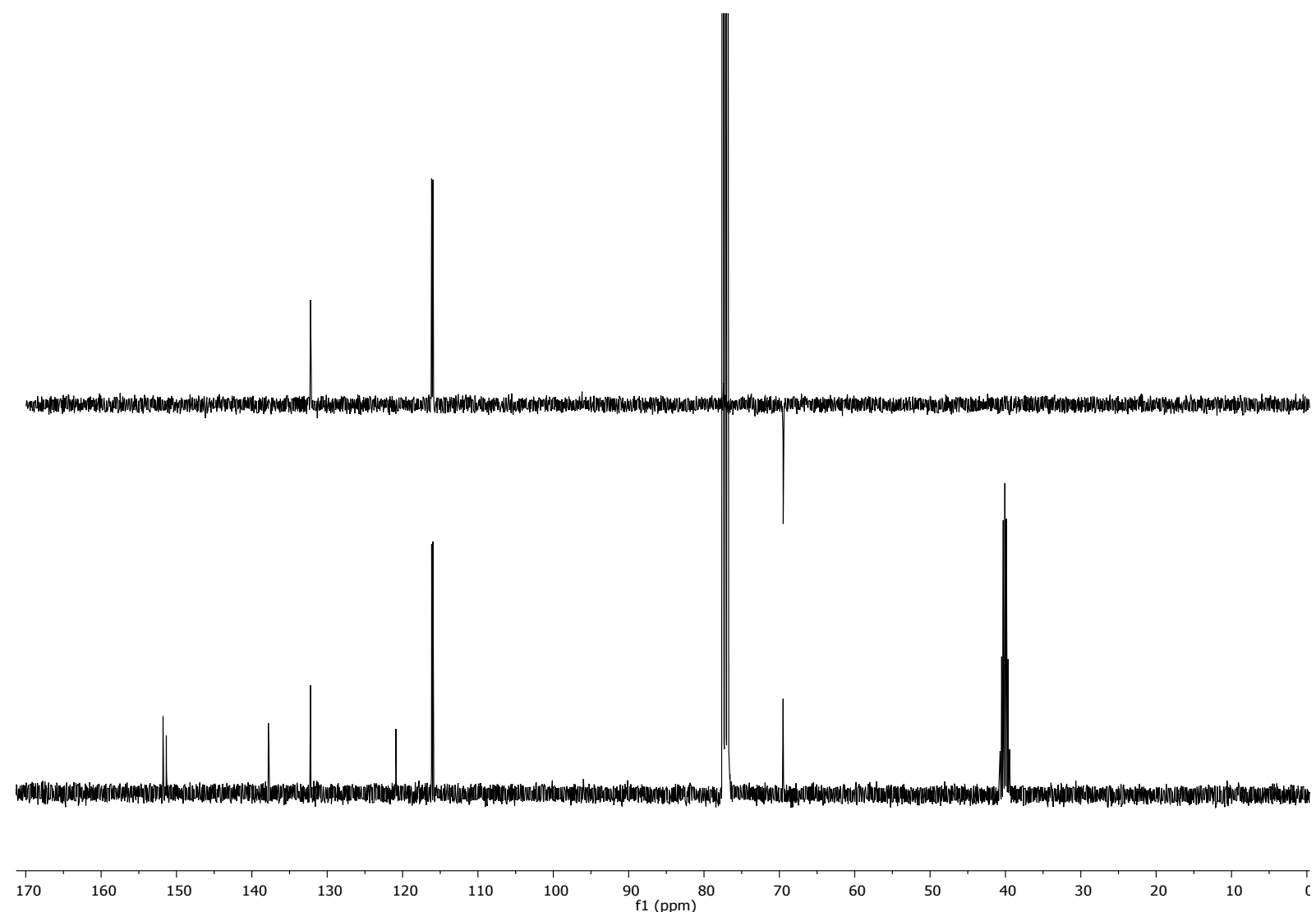

Figure S10. ${ }^{13} \mathrm{C}$ NMR spectrum of compound $3\left(100 \mathrm{MHz}, \mathrm{CDCl}_{3}\right.$ and a drop of DMSO, $298 \mathrm{~K}$ ).

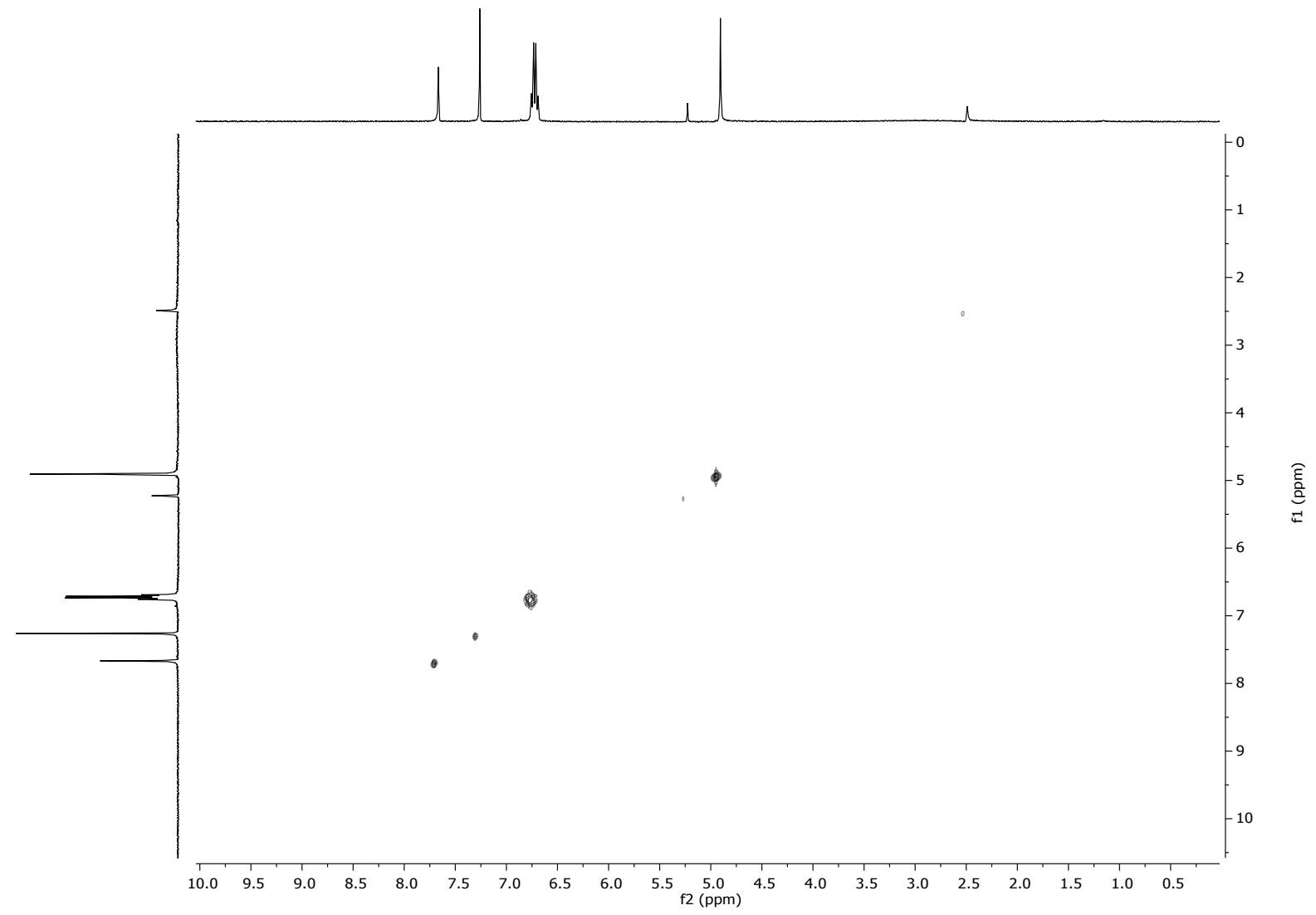

Figure S11. 2D COSY spectrum of compound $3\left(400 \mathrm{MHz}, \mathrm{CDCl}_{3}\right.$ and a drop of DMSO, $298 \mathrm{~K}$ ). 


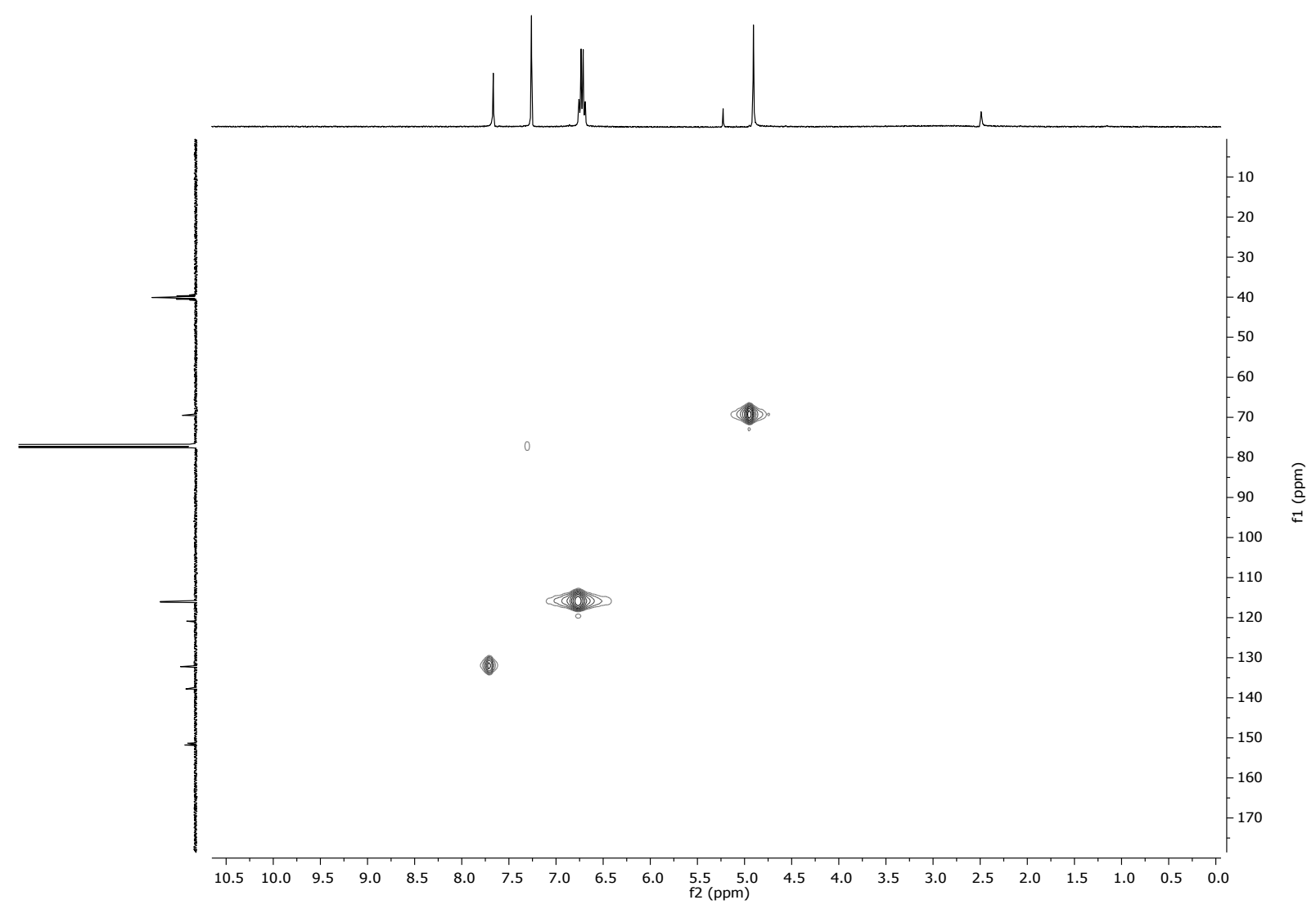

Figure S12. 2D HSQC spectrum of compound $3\left(100 \mathrm{MHz}, \mathrm{CDCl}_{3}\right.$ and a drop of DMSO, $298 \mathrm{~K}$ ).

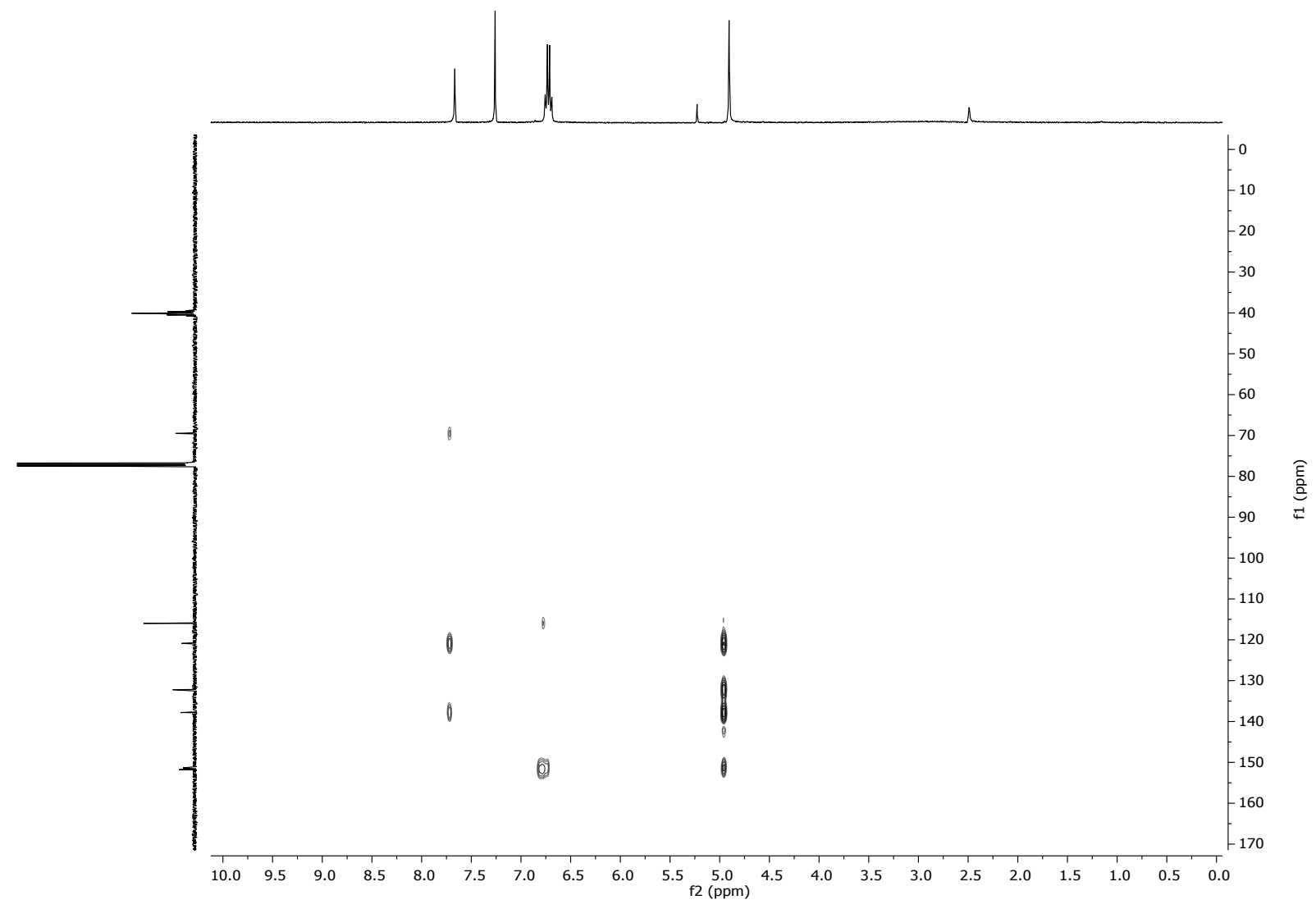

Figure S13. 2D HMBC spectrum of compound $3\left(100 \mathrm{MHz}, \mathrm{CDCl}_{3}\right.$ and a drop of DMSO, $298 \mathrm{~K}$ ). 


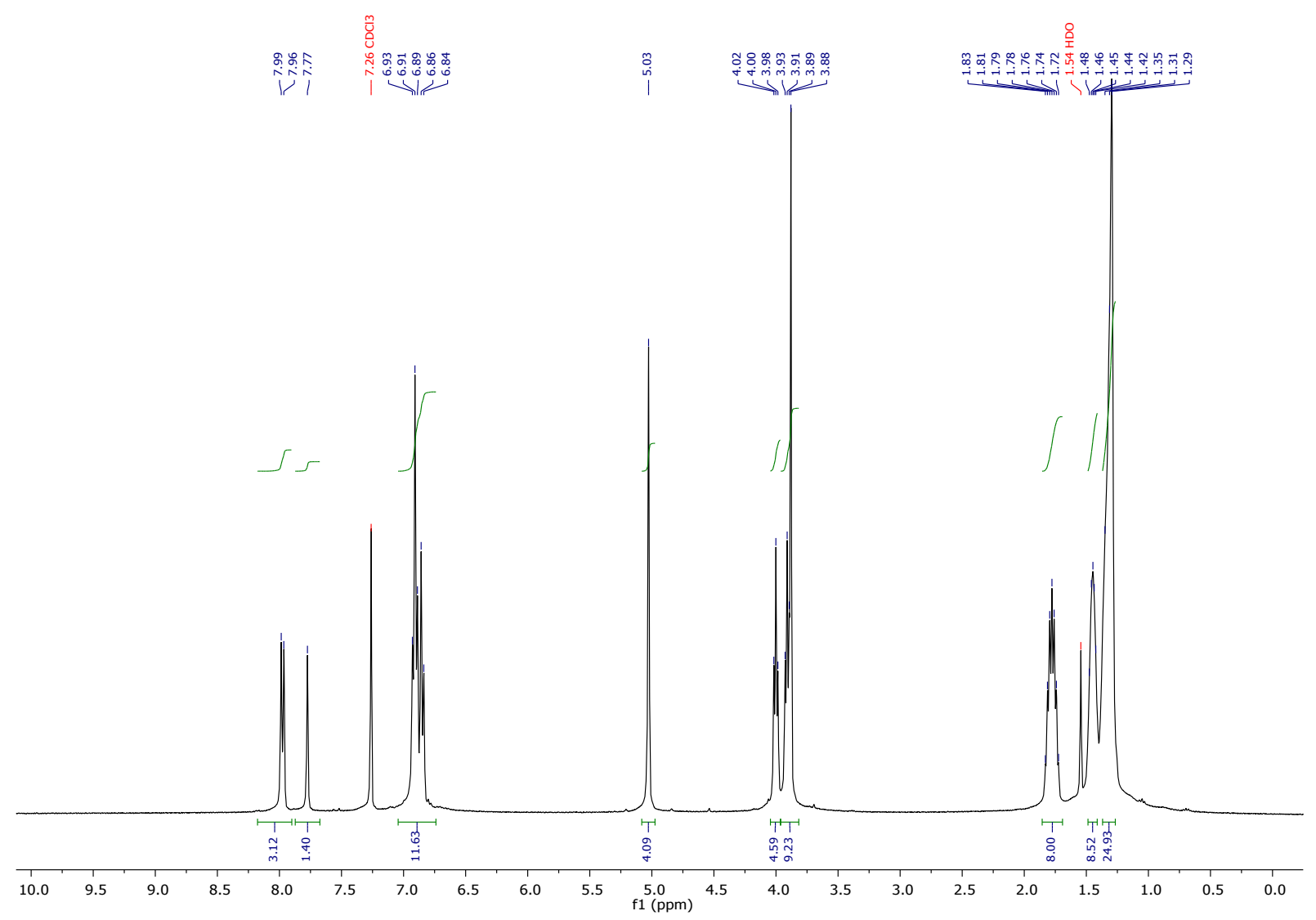

Figure S14. ${ }^{1} \mathrm{H}$ NMR spectrum of compound 5 (400 MHz, $\mathrm{CDCl}_{3}, 298 \mathrm{~K}$ ).
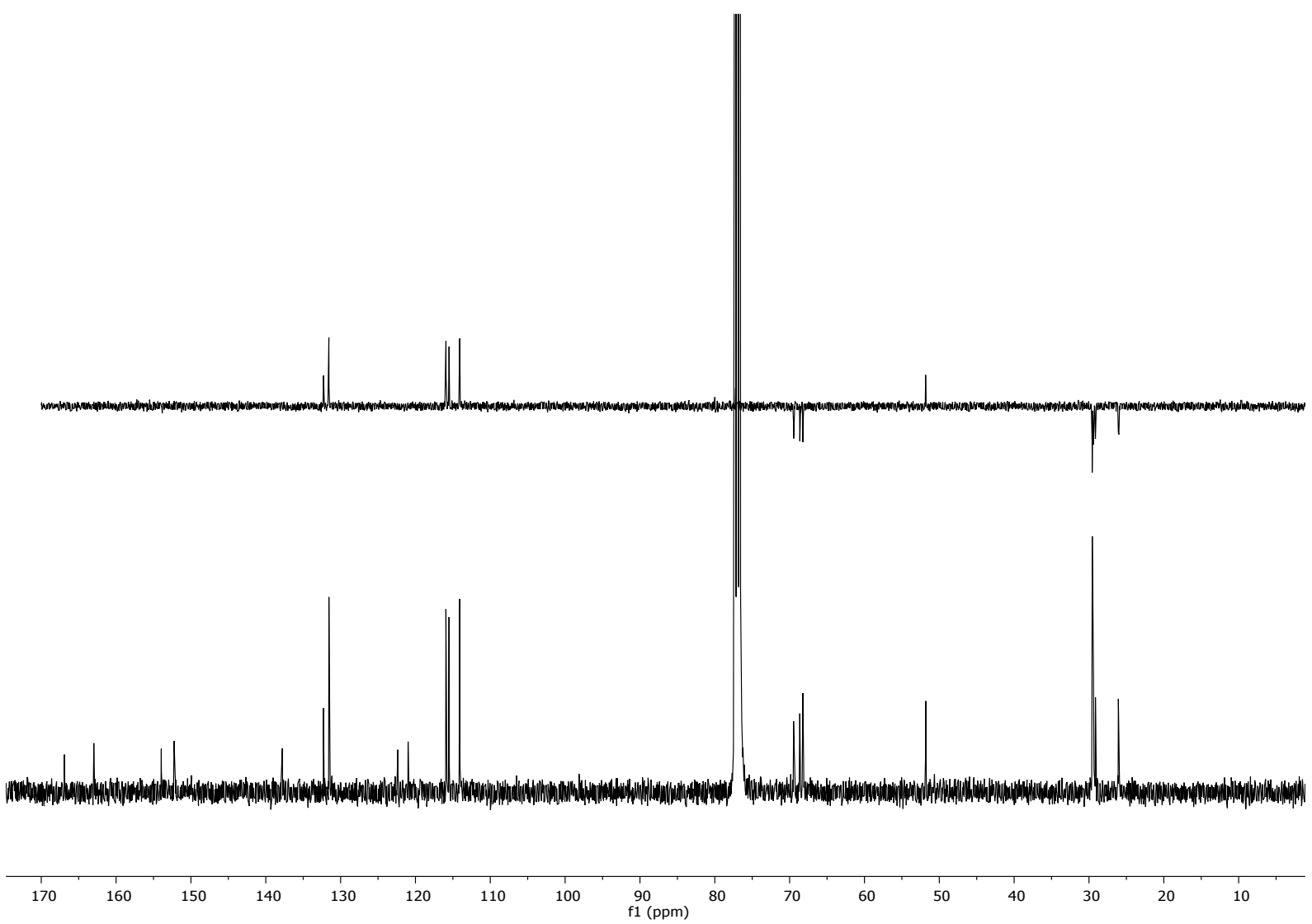

Figure S15. DEPT and ${ }^{13} \mathrm{C}$ NMR spectrum of compound $5\left(100 \mathrm{MHz}, \mathrm{CDCl}_{3}, 298 \mathrm{~K}\right)$. 


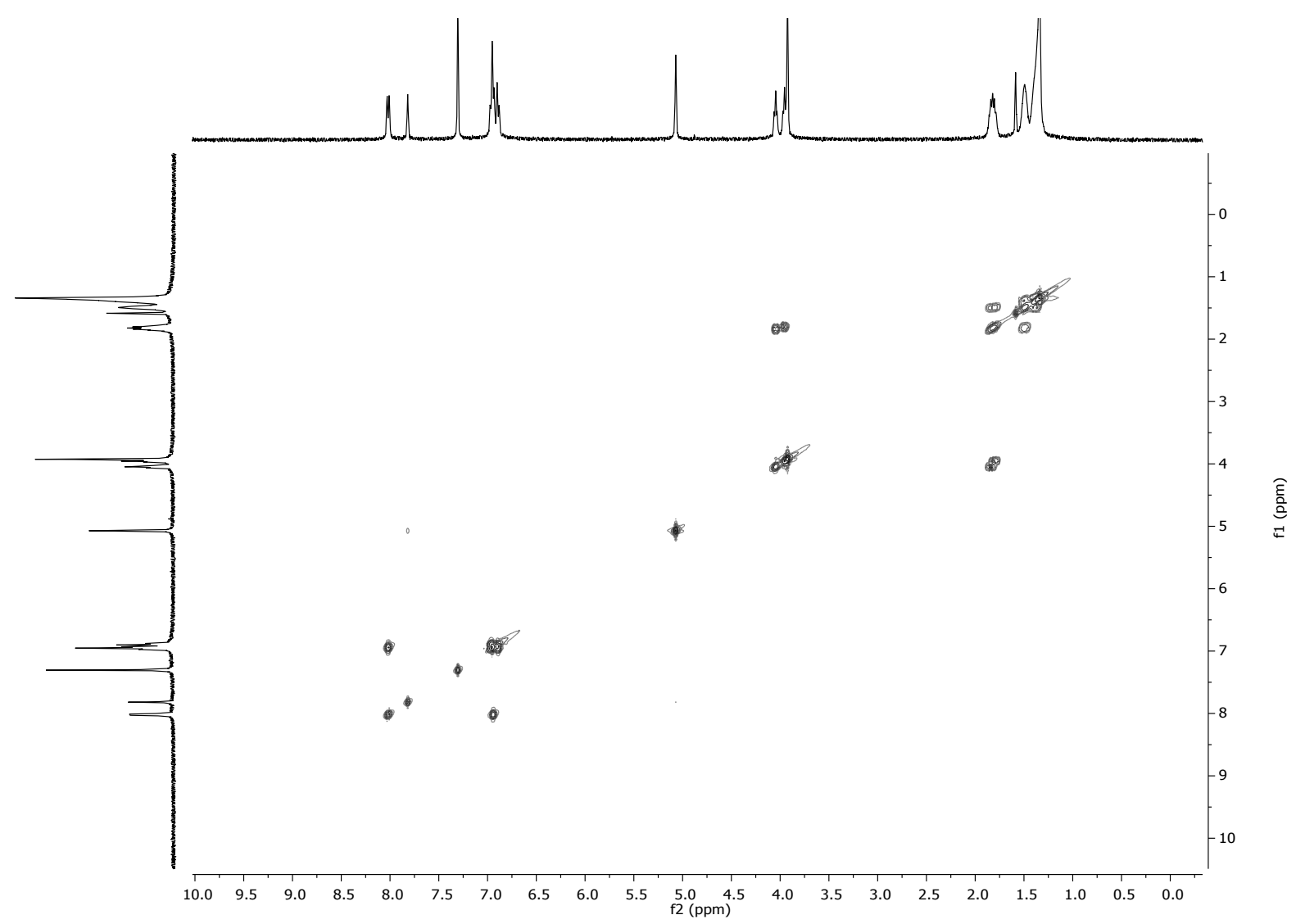

Figure S16. 2D COSY spectrum of compound 5 (400 MHz, CDCl $3,298 \mathrm{~K}$ ).

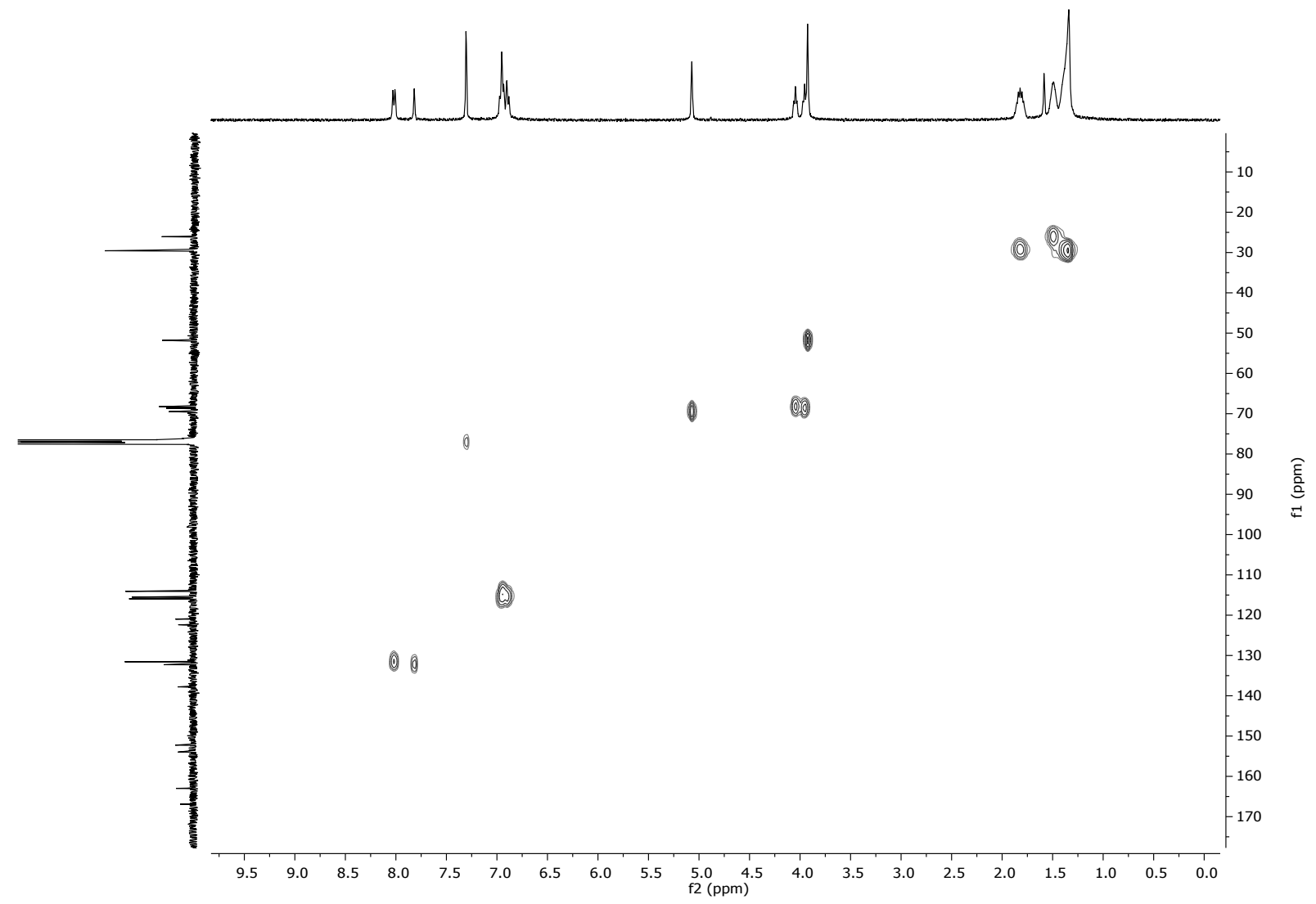

Figure S17. 2D HSQC spectrum of compound $5\left(100 \mathrm{MHz}, \mathrm{CDCl}_{3}, 298 \mathrm{~K}\right)$. 


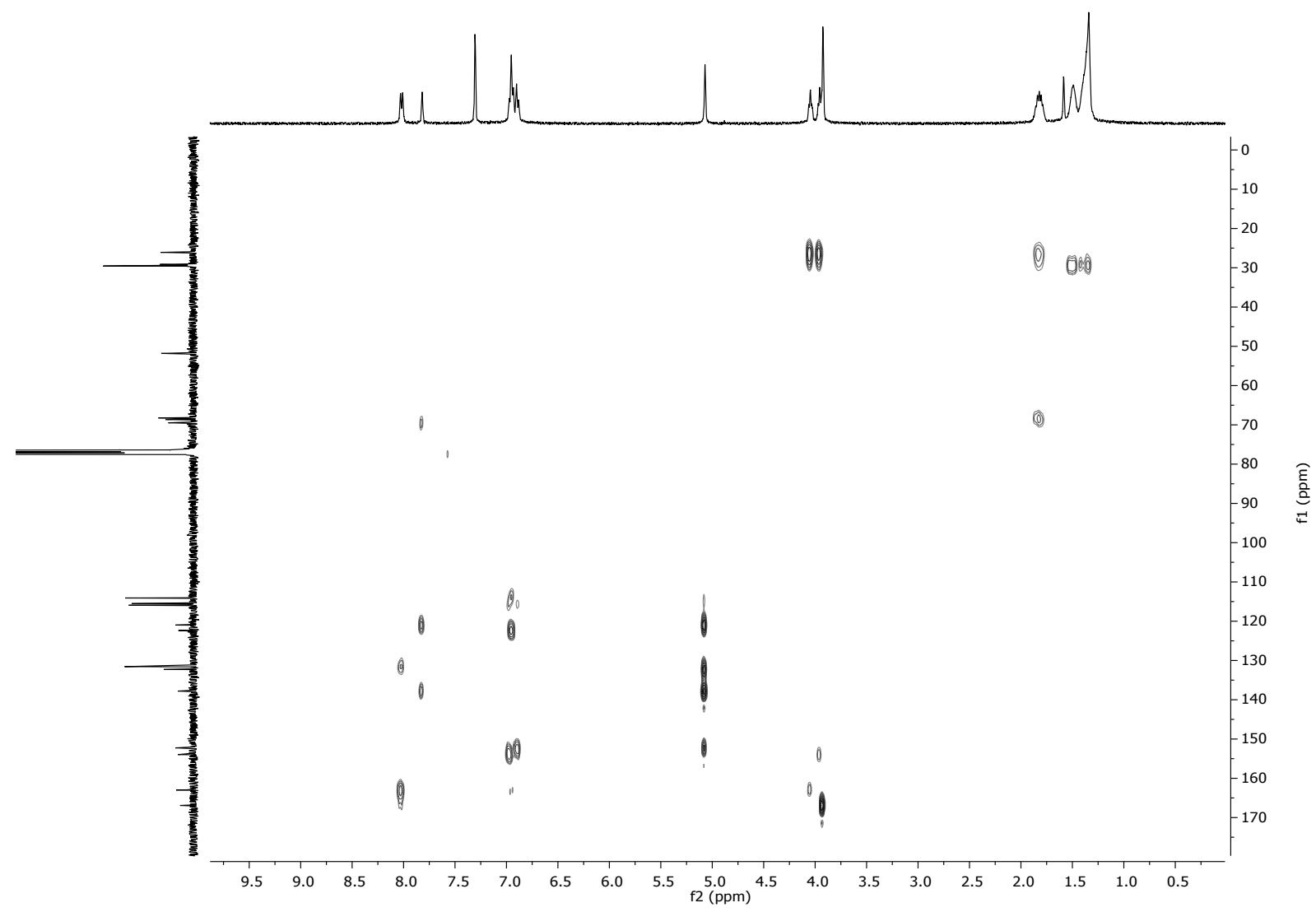

Figure S18. 2D HSQC spectrum of compound 5 (100 MHz, $\mathrm{CDCl}_{3}, 298 \mathrm{~K}$ ).

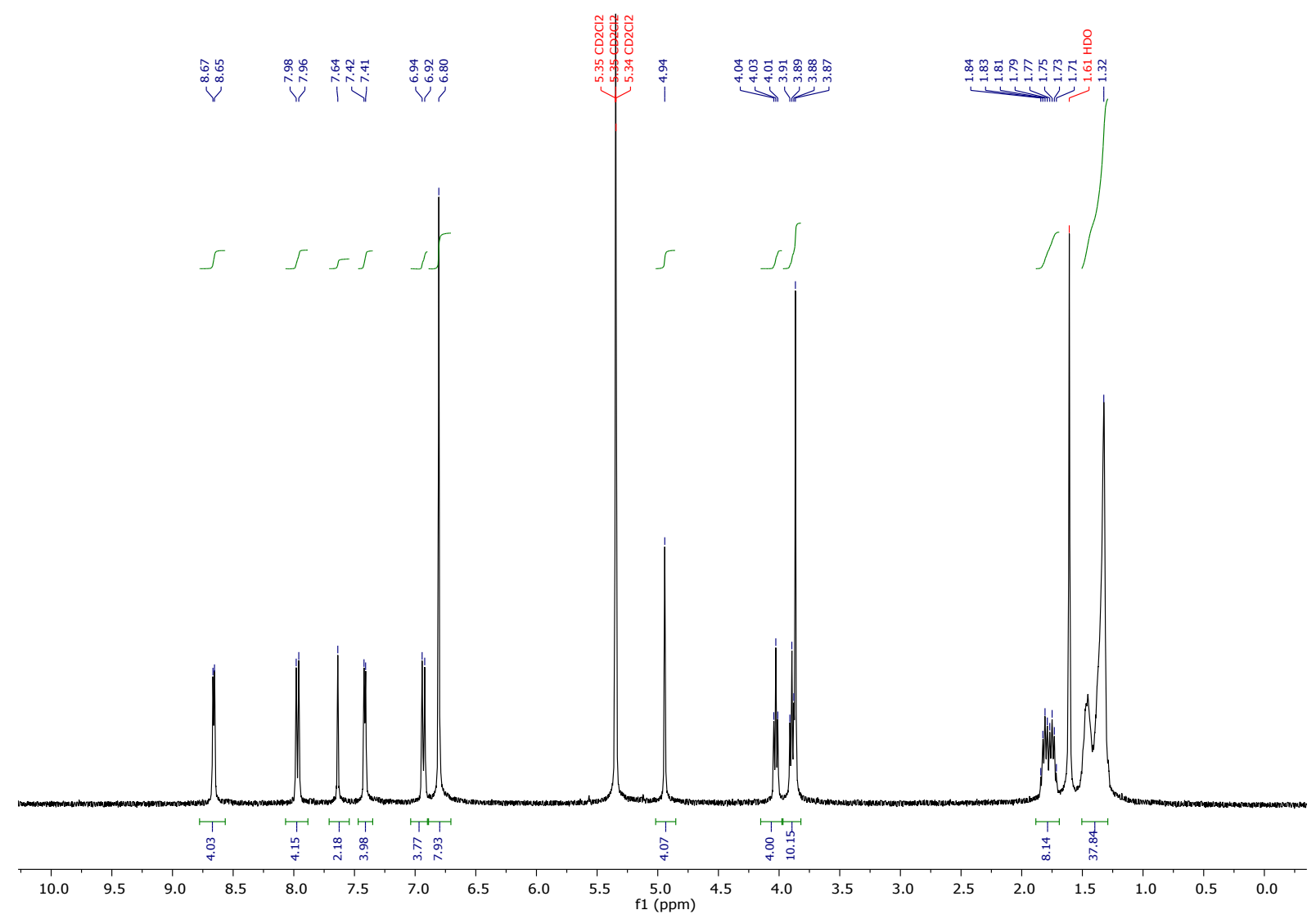

Figure S19. ${ }^{1} \mathrm{H}$ NMR spectrum of compound 6 (400 MHz, $\mathrm{CD}_{2} \mathrm{Cl}_{2}, 298 \mathrm{~K}$ ). 

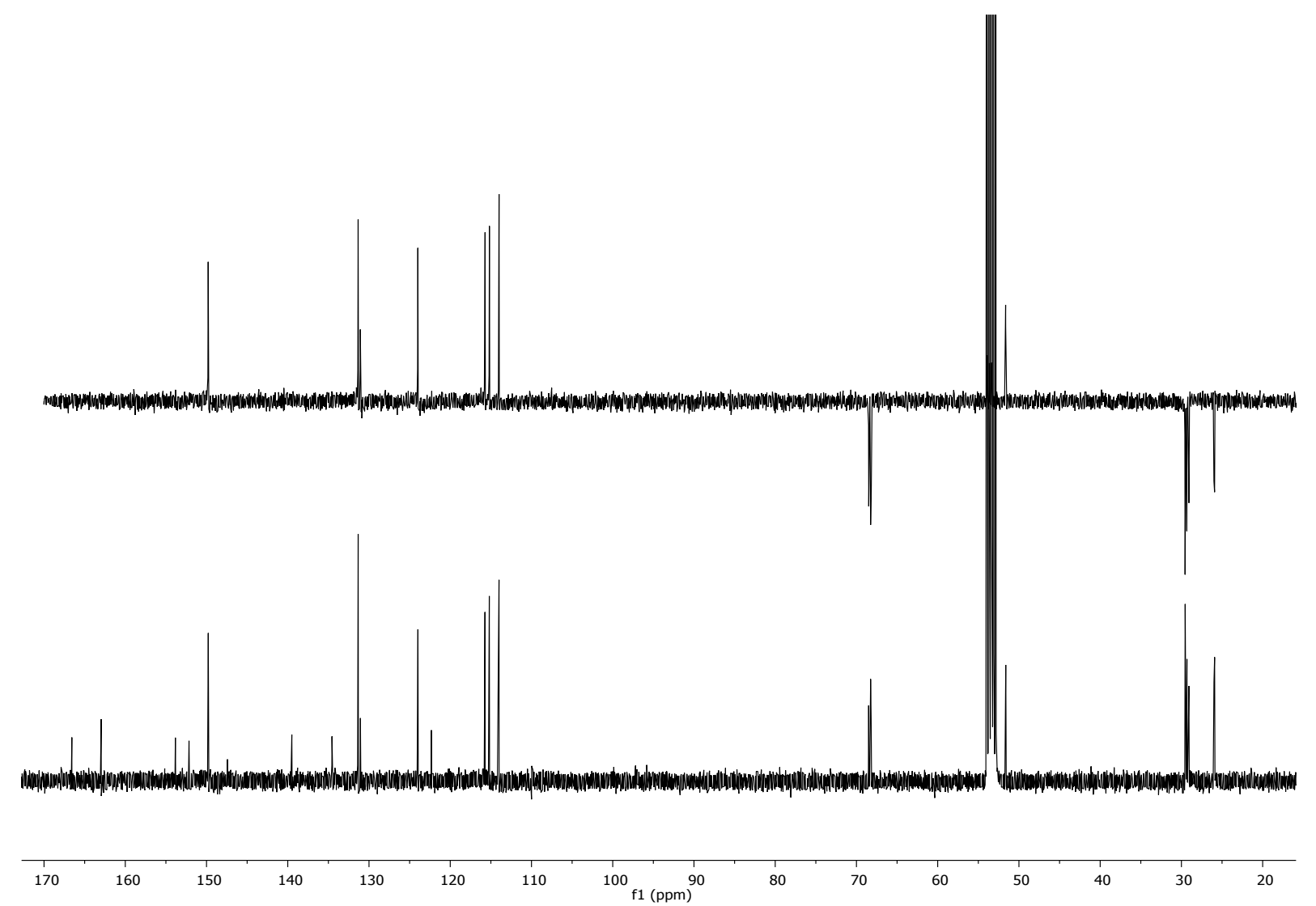

Figure S20. DEPT and ${ }^{13} \mathrm{C}$ NMR spectra of compound $6\left(100 \mathrm{MHz}, \mathrm{CD}_{2} \mathrm{Cl}_{2}, 298 \mathrm{~K}\right)$.

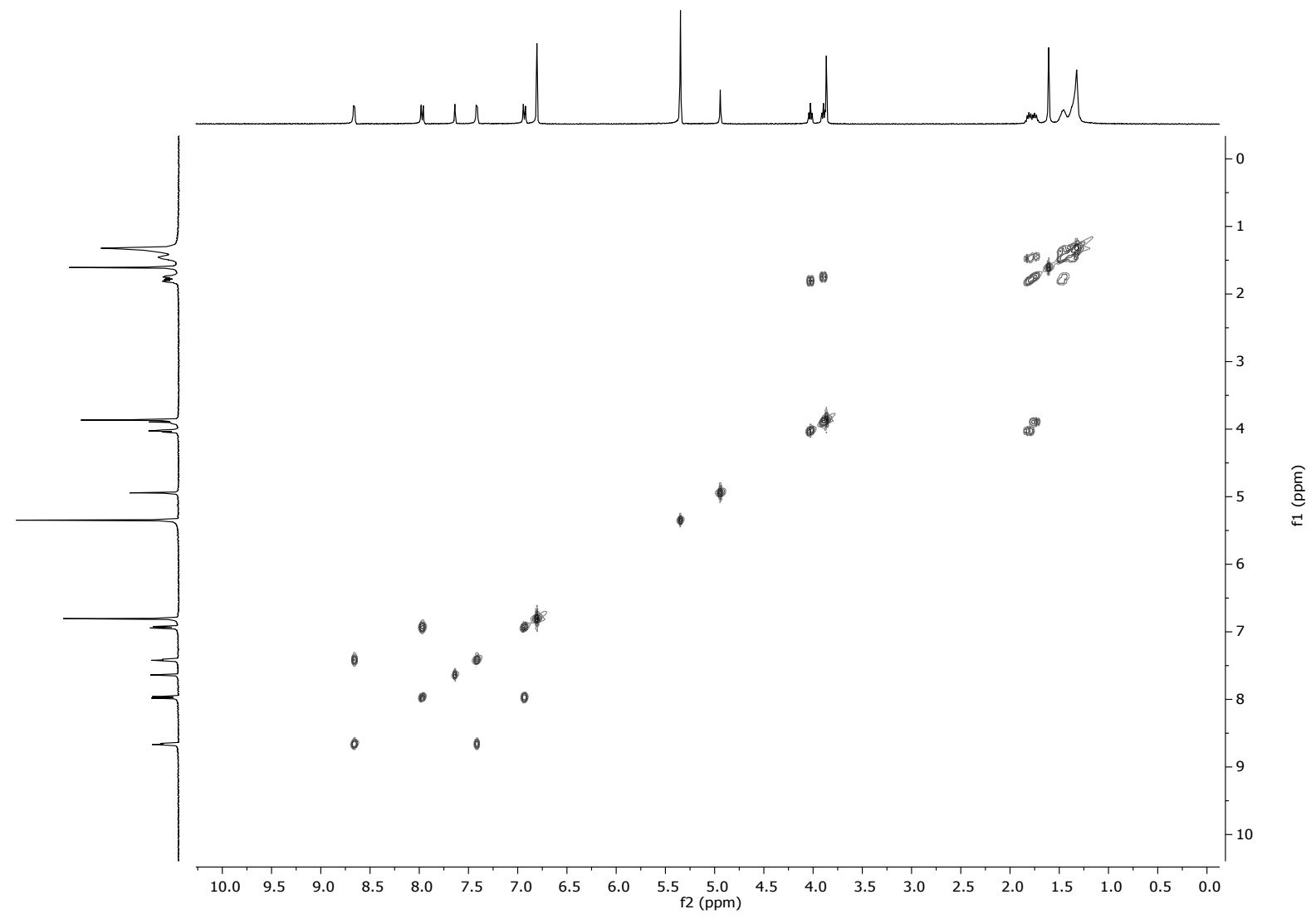

Figure S21. 2D COSY spectrum of compound $6\left(400 \mathrm{MHz}, \mathrm{CD}_{2} \mathrm{Cl}_{2}, 298 \mathrm{~K}\right)$. 


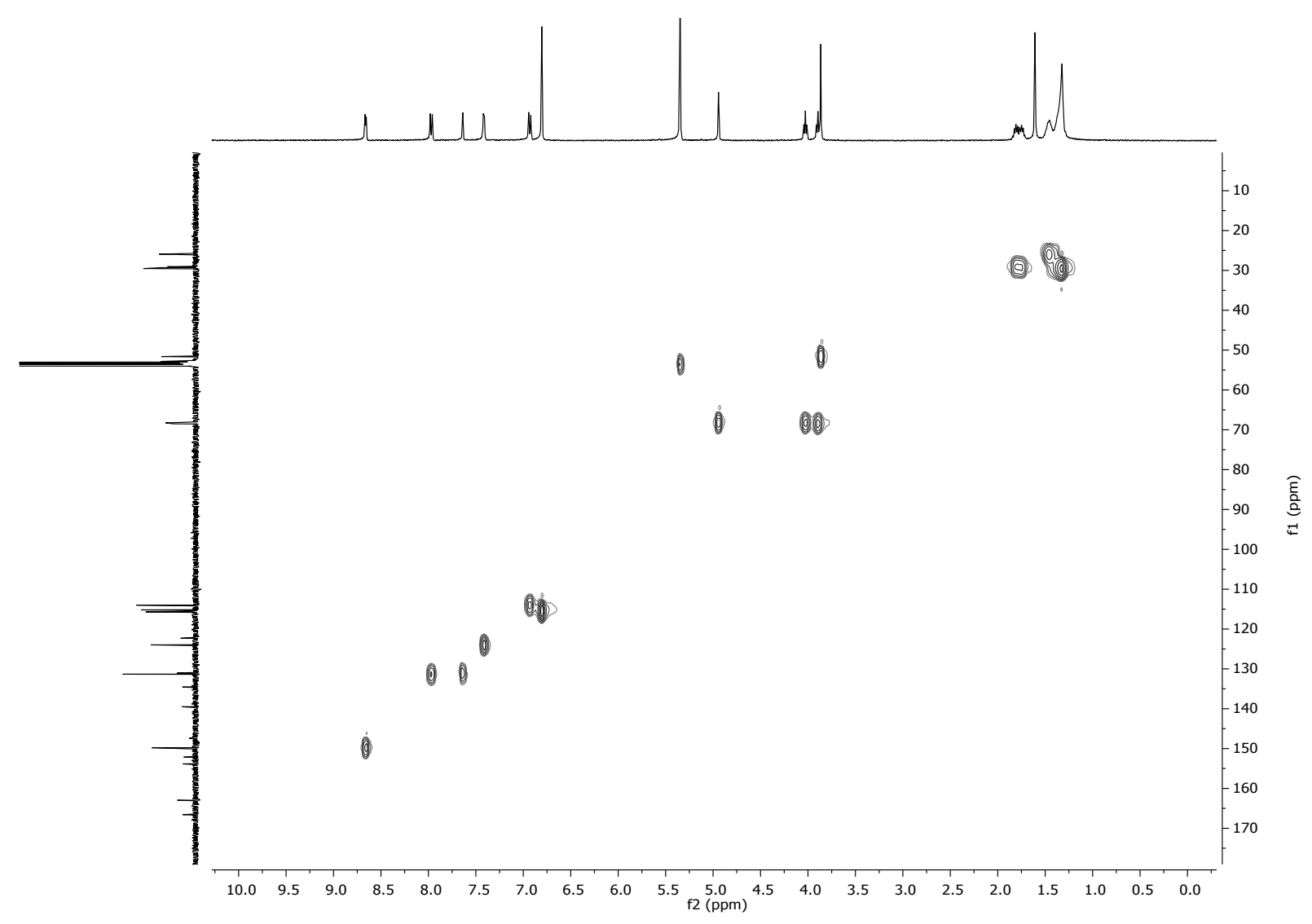

Figure S22. 2D HSQC spectrum of compound 6 (100 MHz, $\left.\mathrm{CD}_{2} \mathrm{Cl}_{2}, 298 \mathrm{~K}\right)$.

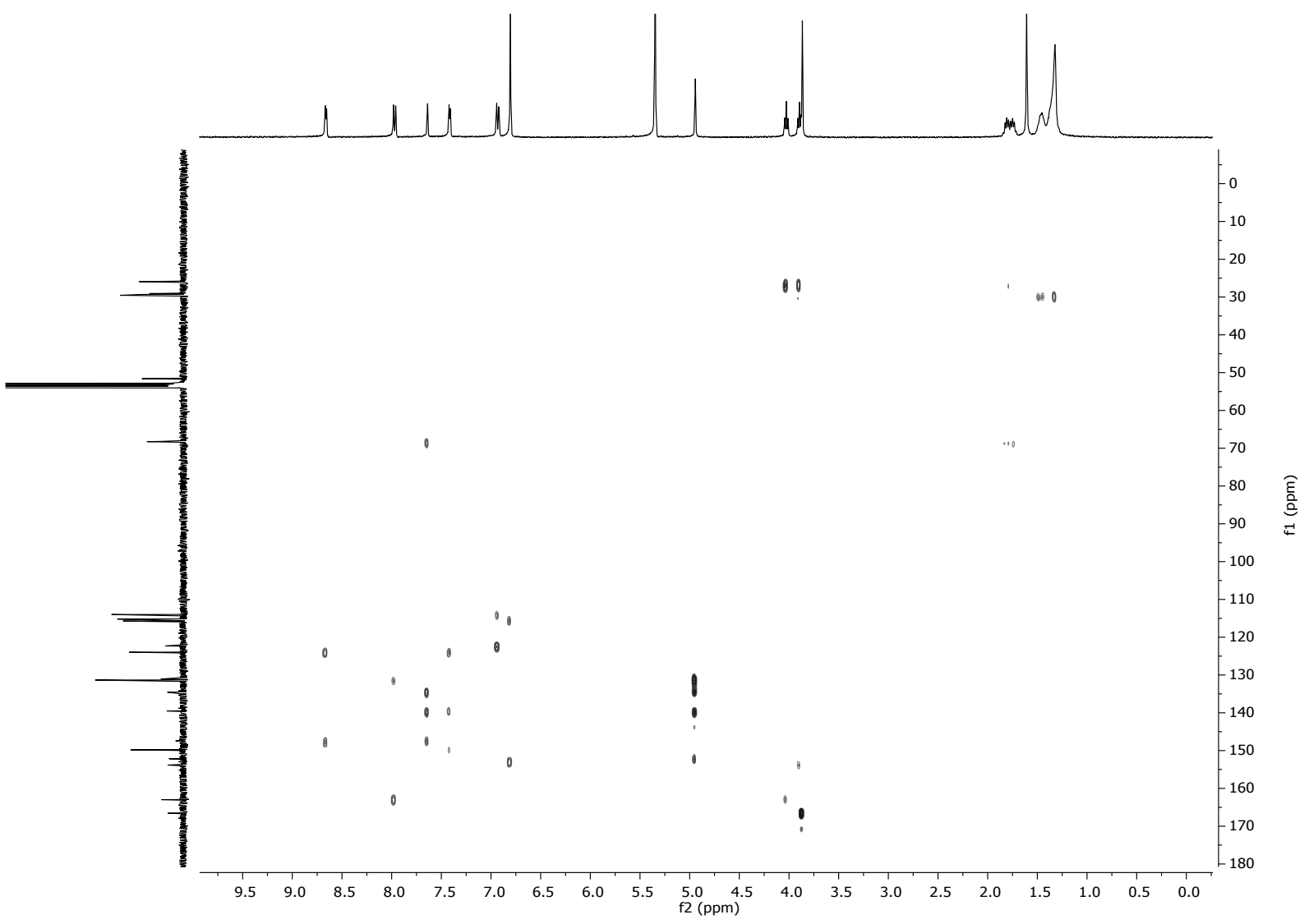

Figure S23. 2D HMBC spectrum of compound 6 (100 MHz, $\left.\mathrm{CD}_{2} \mathrm{Cl}_{2}, 298 \mathrm{~K}\right)$. 


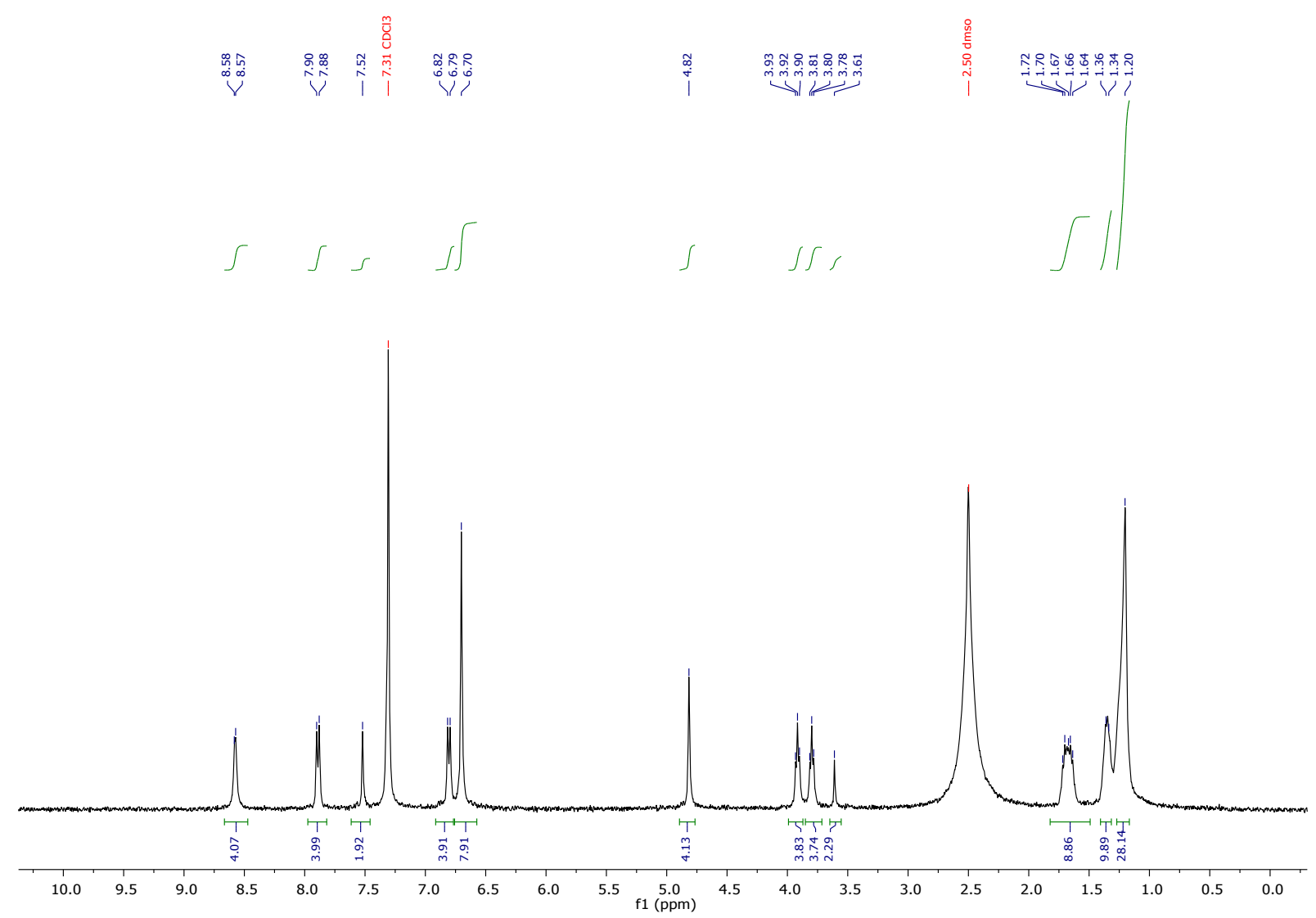

Figure S24. ${ }^{1} \mathrm{H}$ NMR spectrum of compound 7 (400 MHz, $\mathrm{CDCl}_{3}$ and a drop of DMSO, $298 \mathrm{~K}$ ).
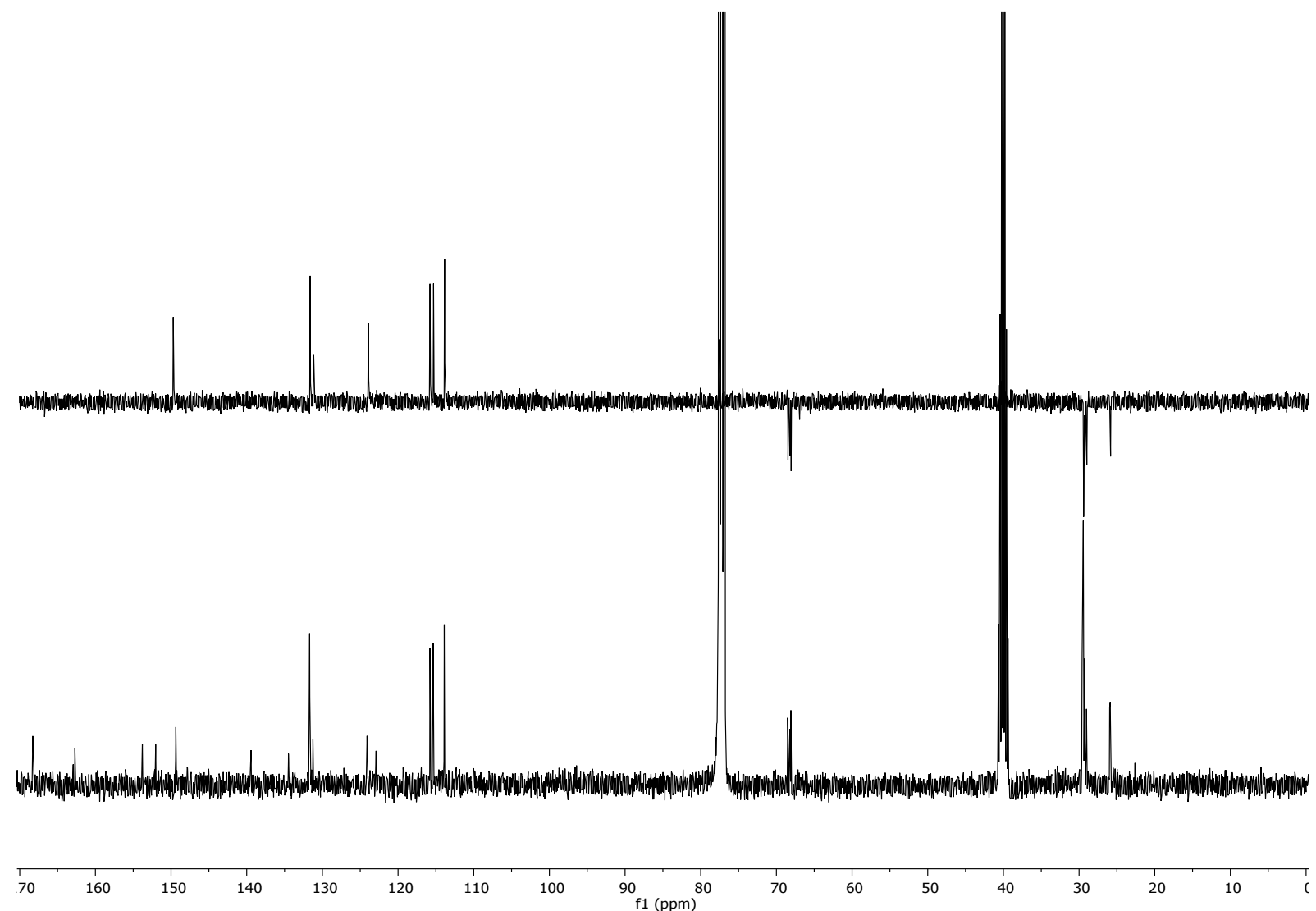

Figure S25. DEPT and ${ }^{13} \mathrm{C}$ NMR spectra of compound 7 ( $100 \mathrm{MHz}, \mathrm{CDCl}_{3}$ and a drop of DMSO, $298 \mathrm{~K}$ ). 


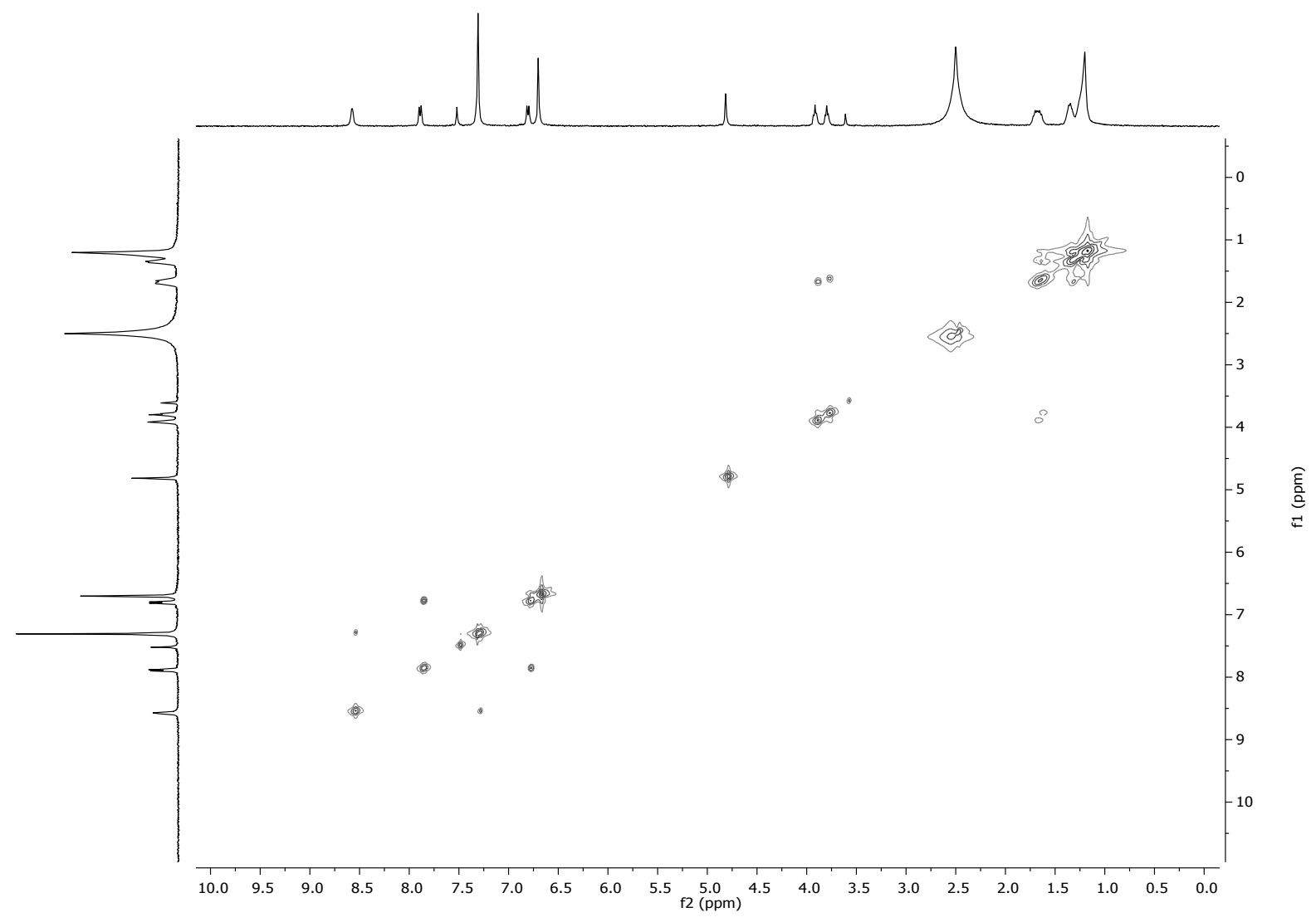

Figure S26. 2D COSY spectrum of compound 7 ( $400 \mathrm{MHz}^{\mathrm{CDCl}} \mathrm{CDC}_{3}$ and a drop of DMSO, $298 \mathrm{~K}$ ).

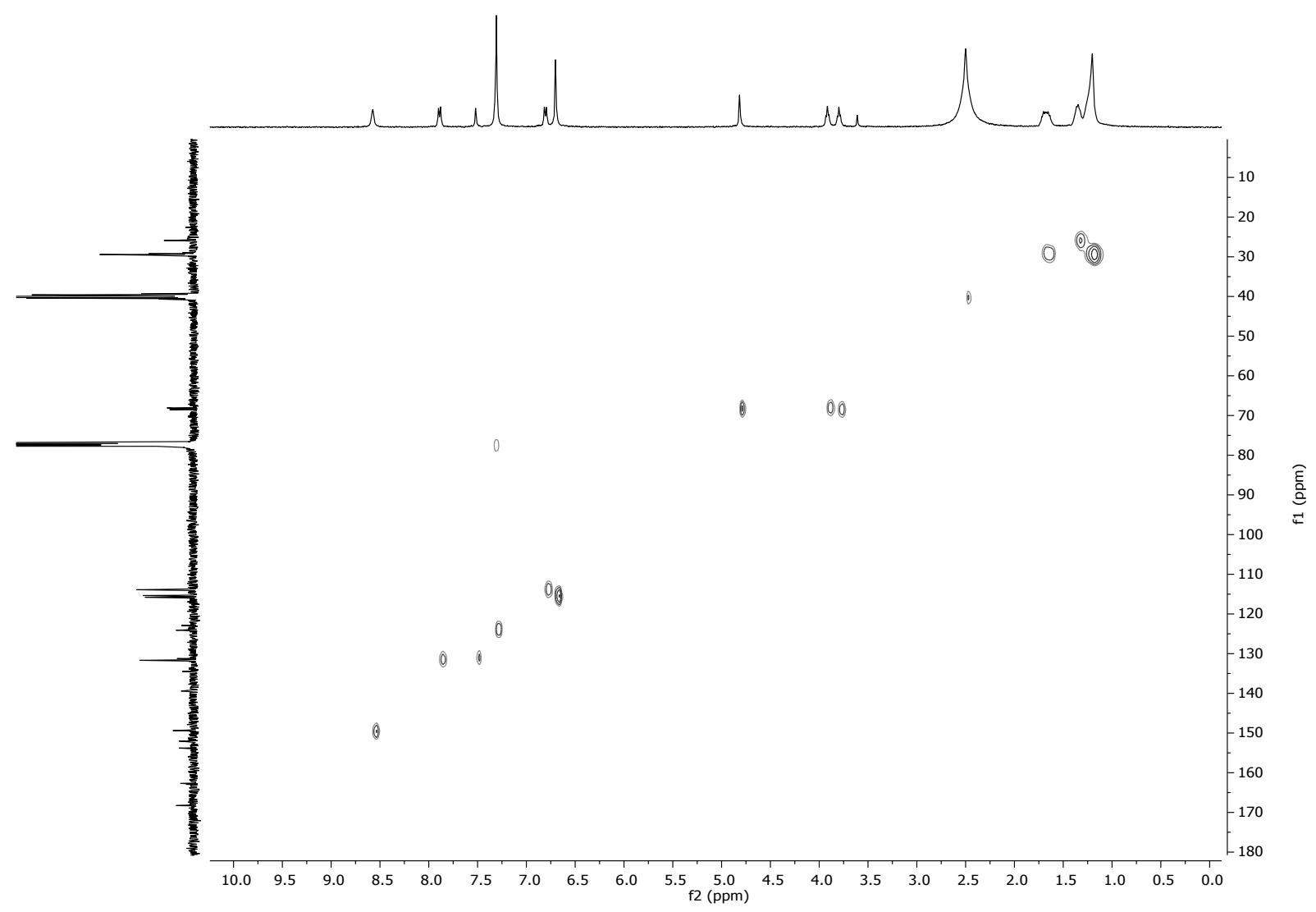

Figure S27. 2D HSQC spectrum of compound $7\left(100 \mathrm{MHz}, \mathrm{CDCl}_{3}\right.$ and a drop of DMSO, $\left.298 \mathrm{~K}\right)$. 


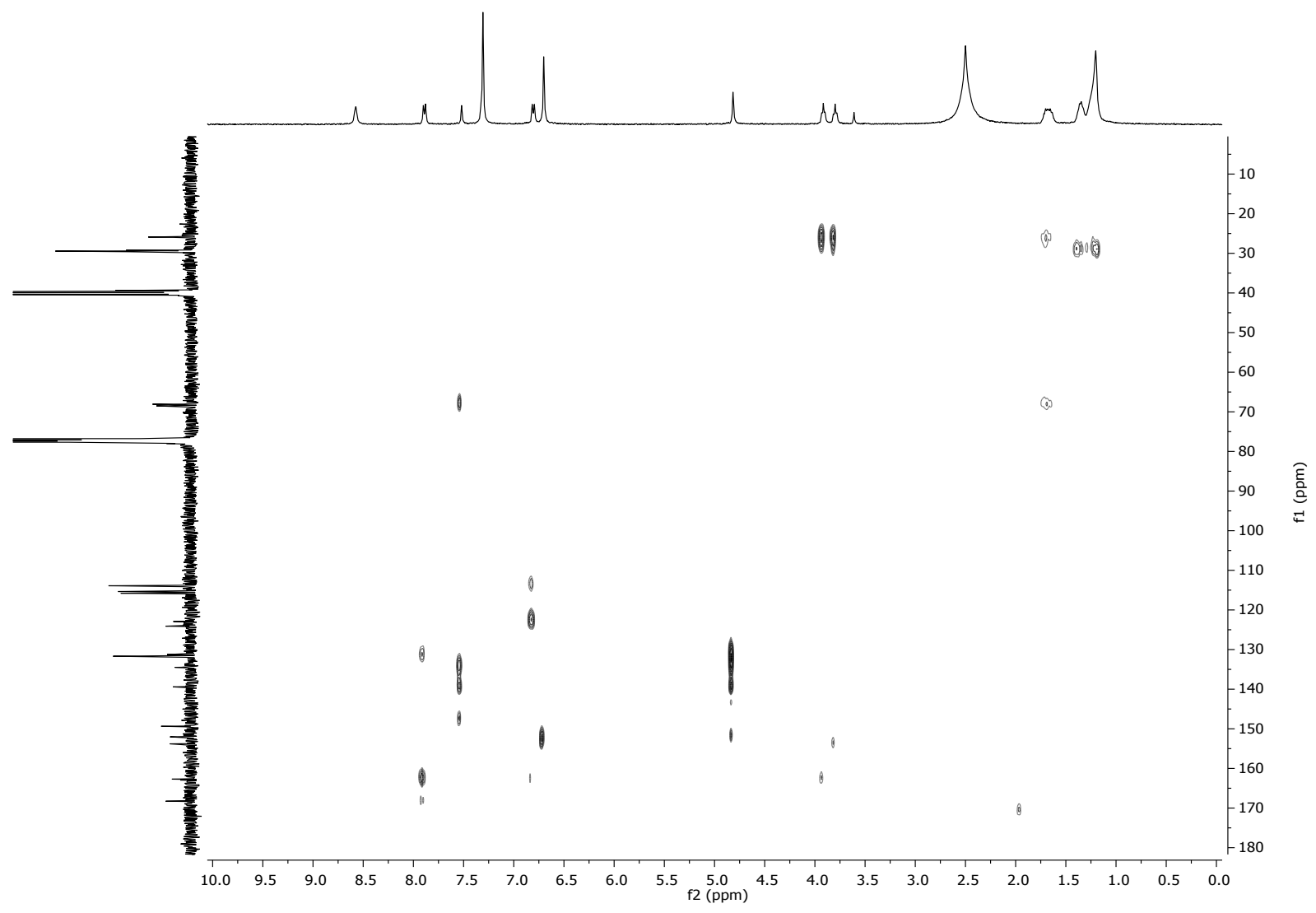

Figure S28. 2D HMBC spectrum of compound 7 (100 MHz, CDCl 3 and a drop of DMSO, $298 \mathrm{~K}$ ).

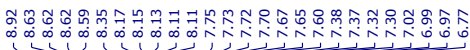

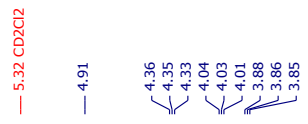

$$
-r, \int d i d s
$$

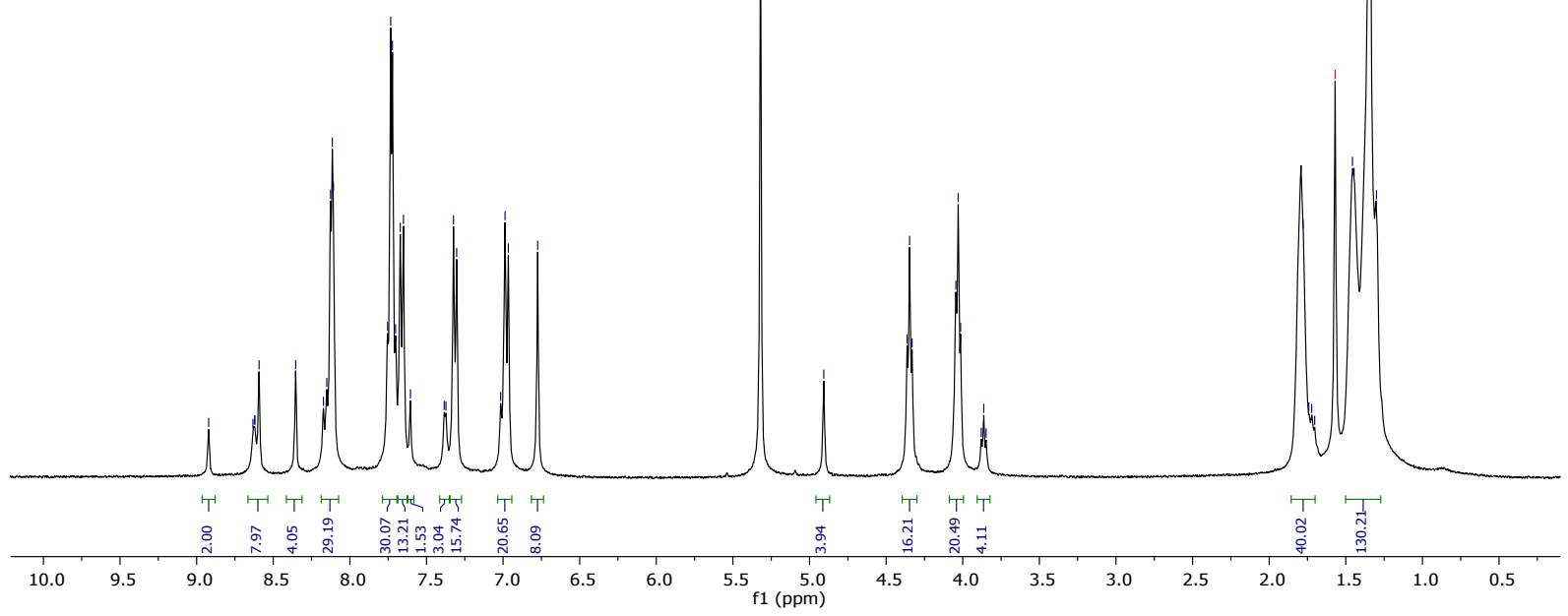

Figure S29. ${ }^{1} \mathrm{H}$ NMR spectrum of $\mathbf{L}\left(400 \mathrm{MHz}, \mathrm{CD}_{2} \mathrm{Cl}_{2}, 298 \mathrm{~K}\right)$.

$\mathrm{S} 21$ 

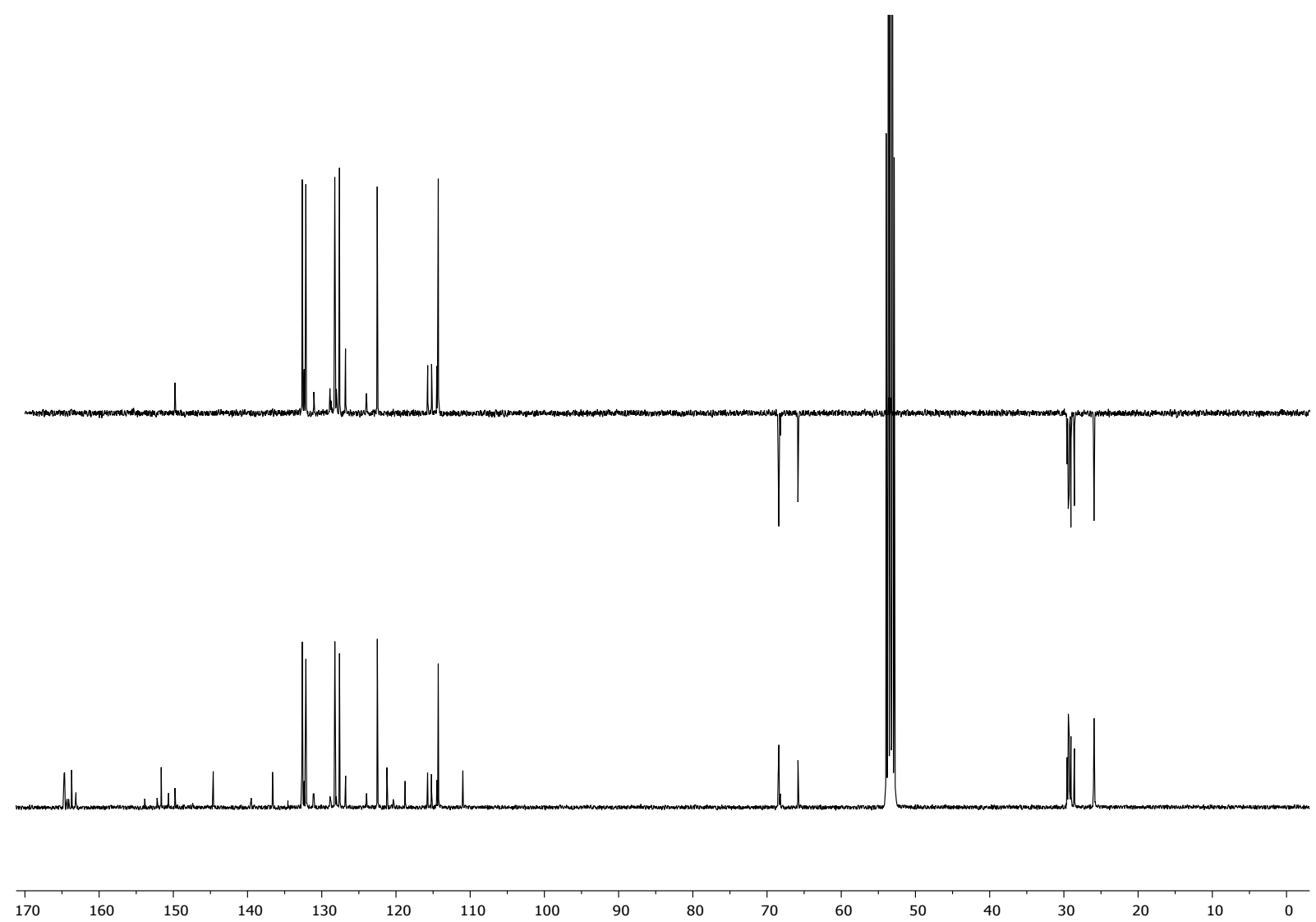

Figure S30. DEPT and ${ }^{13} \mathrm{C}$ NMR spectra of $\mathrm{L}\left(100 \mathrm{MHz}, \mathrm{CD}_{2} \mathrm{Cl}_{2}, 298 \mathrm{~K}\right)$.

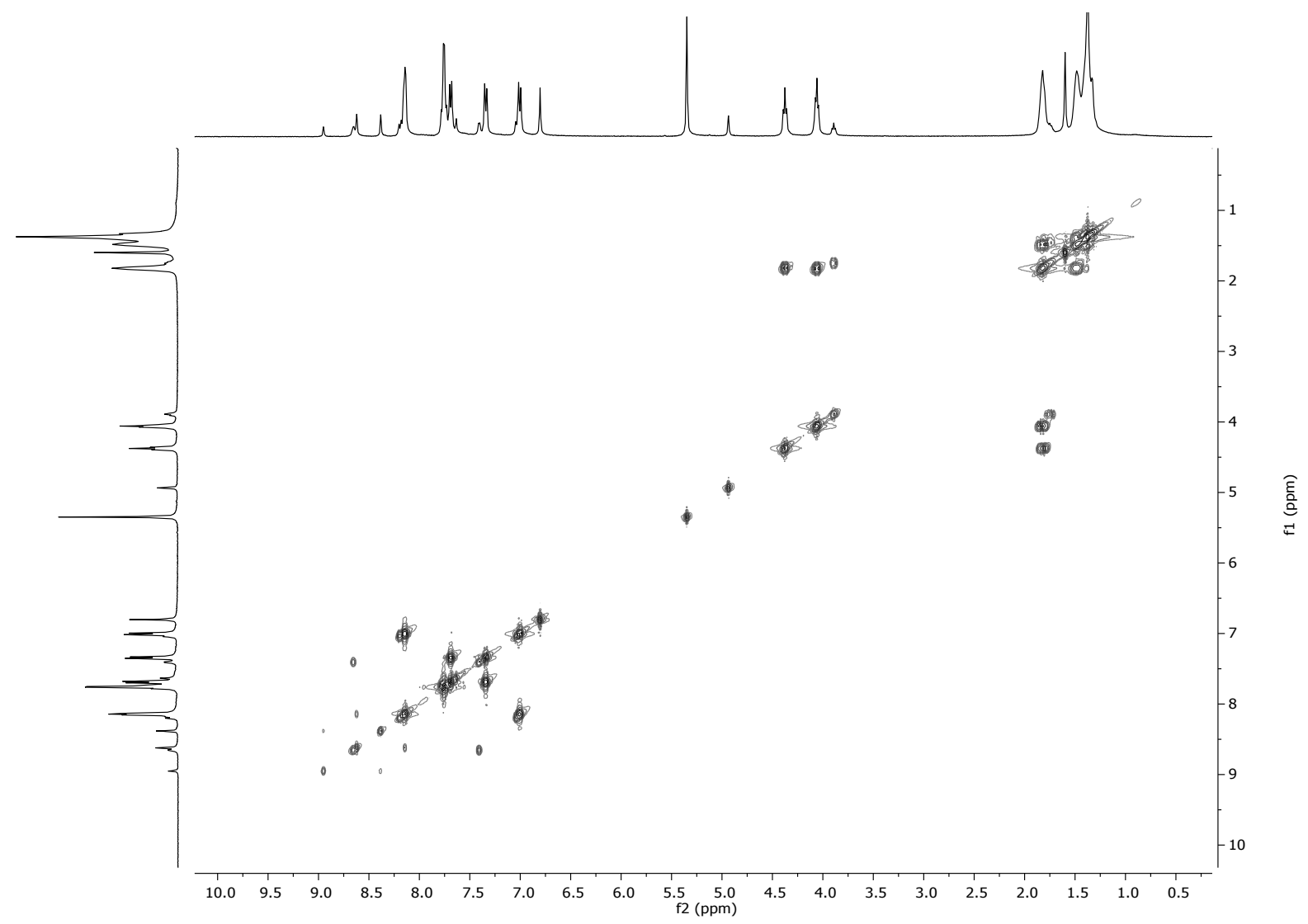

Figure S31. 2D COSY spectrum of $\mathrm{L}\left(400 \mathrm{MHz}, \mathrm{CD}_{2} \mathrm{Cl}_{2}, 298 \mathrm{~K}\right)$. 


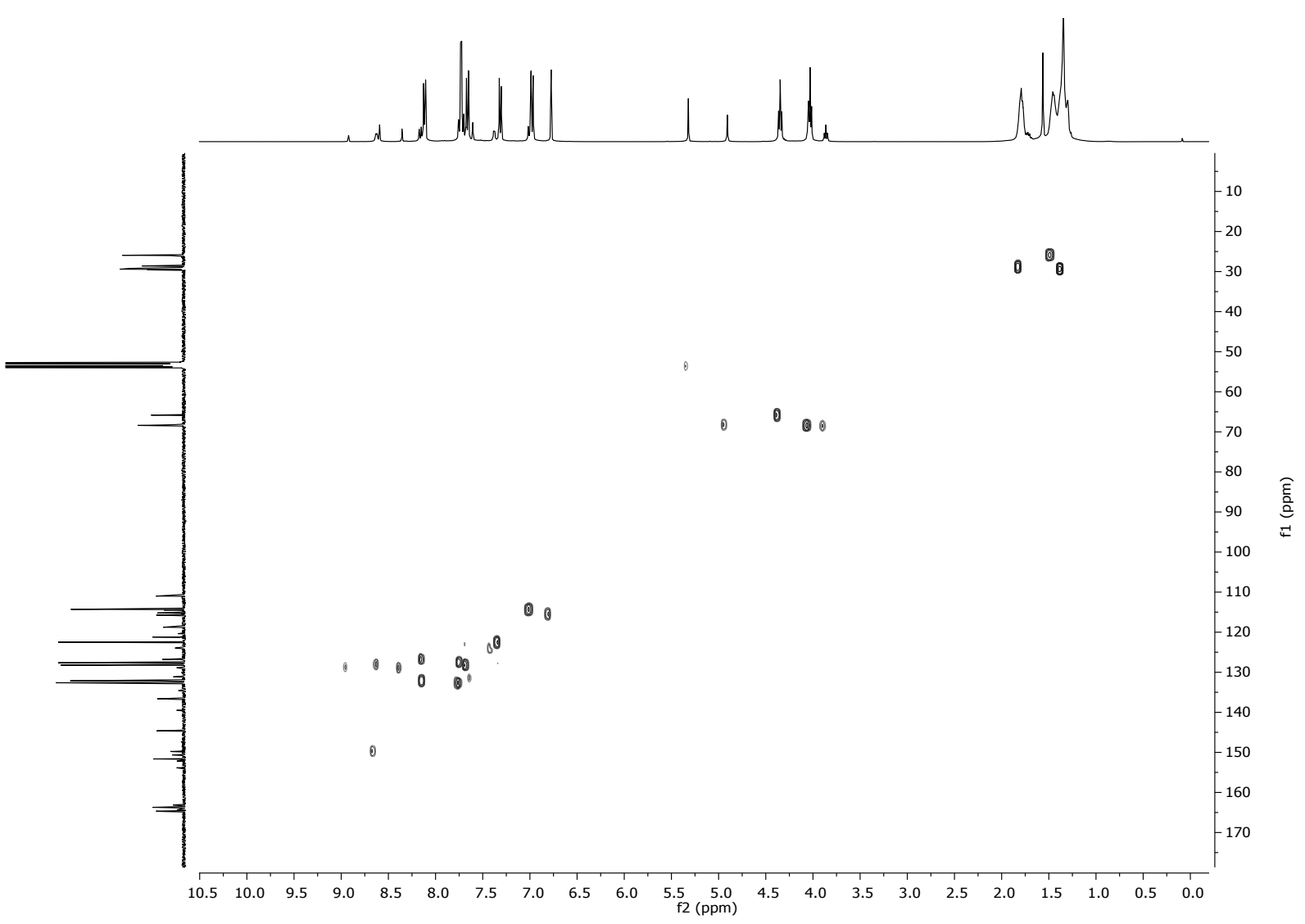

Figure S32. 2D HSQC spectrum of $\mathbf{L}\left(100 \mathrm{MHz}, \mathrm{CD}_{2} \mathrm{Cl}_{2}, 298 \mathrm{~K}\right)$.

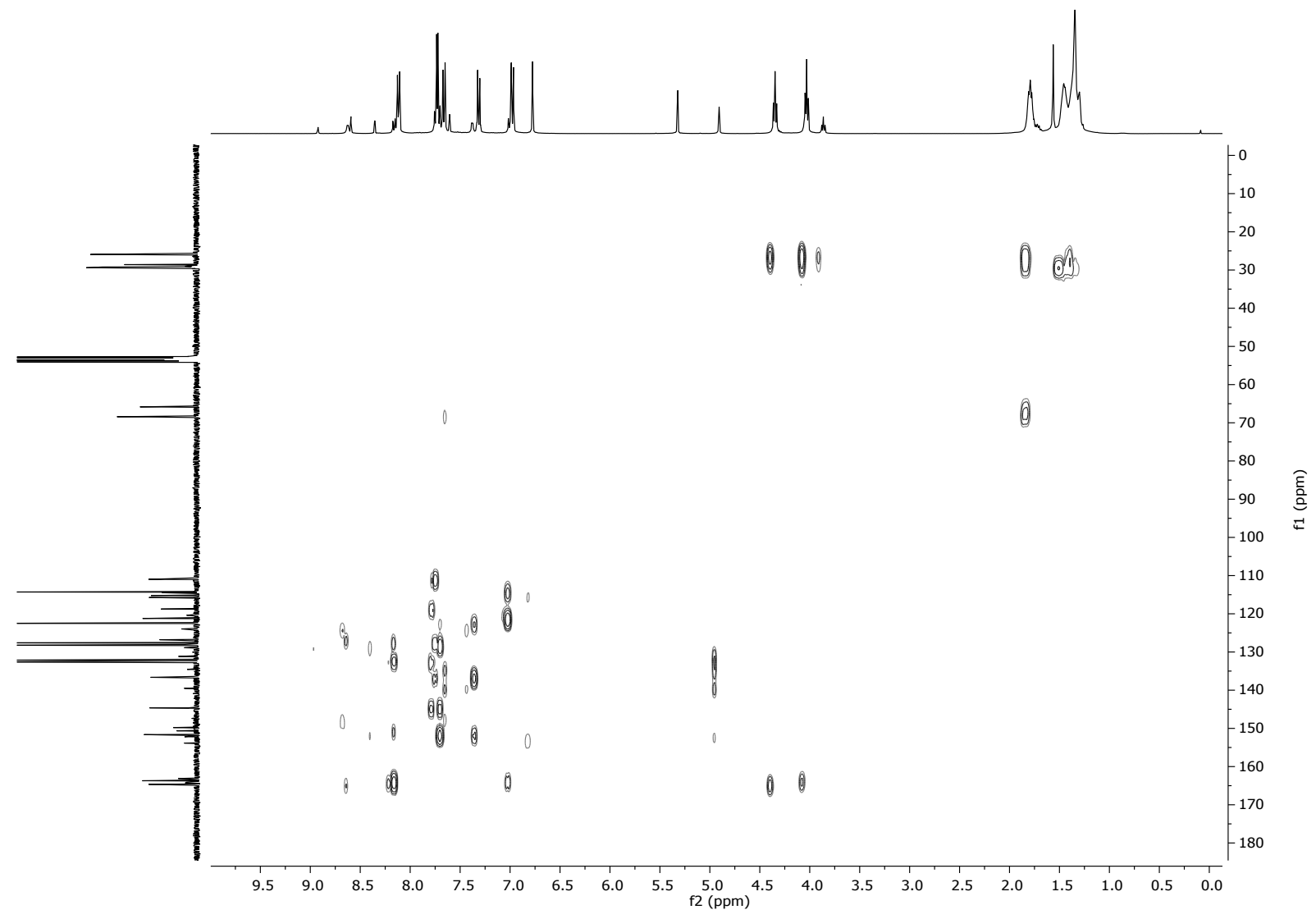

Figure S33. 2D HMBC spectrum of $\mathbf{L}\left(100 \mathrm{MHz}, \mathrm{CD}_{2} \mathrm{Cl}_{2}, 298 \mathrm{~K}\right)$. 


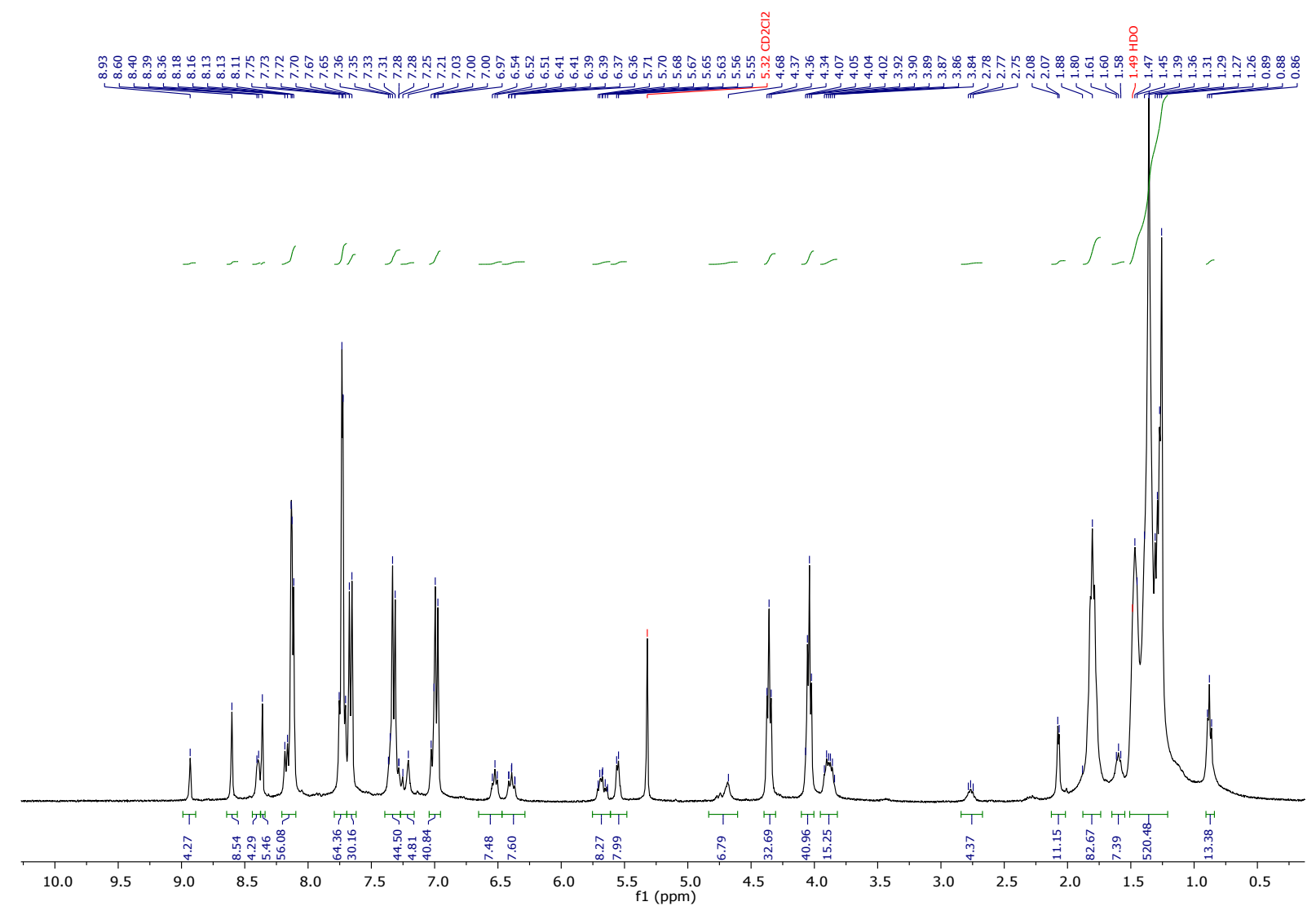

Figure S34. ${ }^{1} \mathrm{H}$ NMR spectrum of $\mathbf{M}_{\mathbf{2}} \mathbf{L}_{2}\left(400 \mathrm{MHz}, \mathrm{CD}_{2} \mathrm{Cl}_{2}, 298 \mathrm{~K}\right)$.
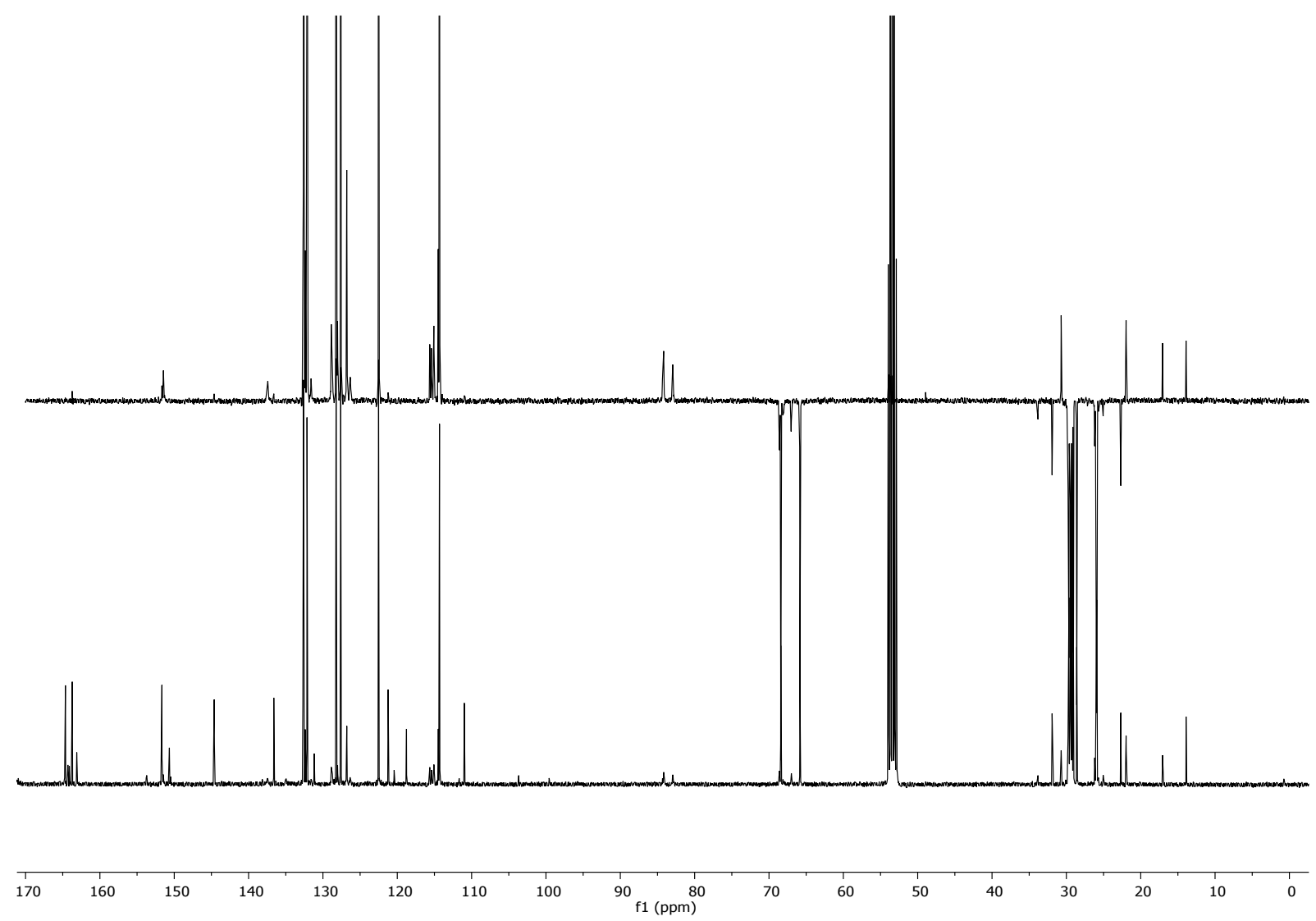

Figure S35. DEPT and ${ }^{13} \mathrm{C}$ NMR spectra of $\mathbf{M}_{\mathbf{2}} \mathbf{L}_{\mathbf{2}}\left(100 \mathrm{MHz}, \mathrm{CD}_{2} \mathrm{Cl}_{2}, 298 \mathrm{~K}\right)$. 


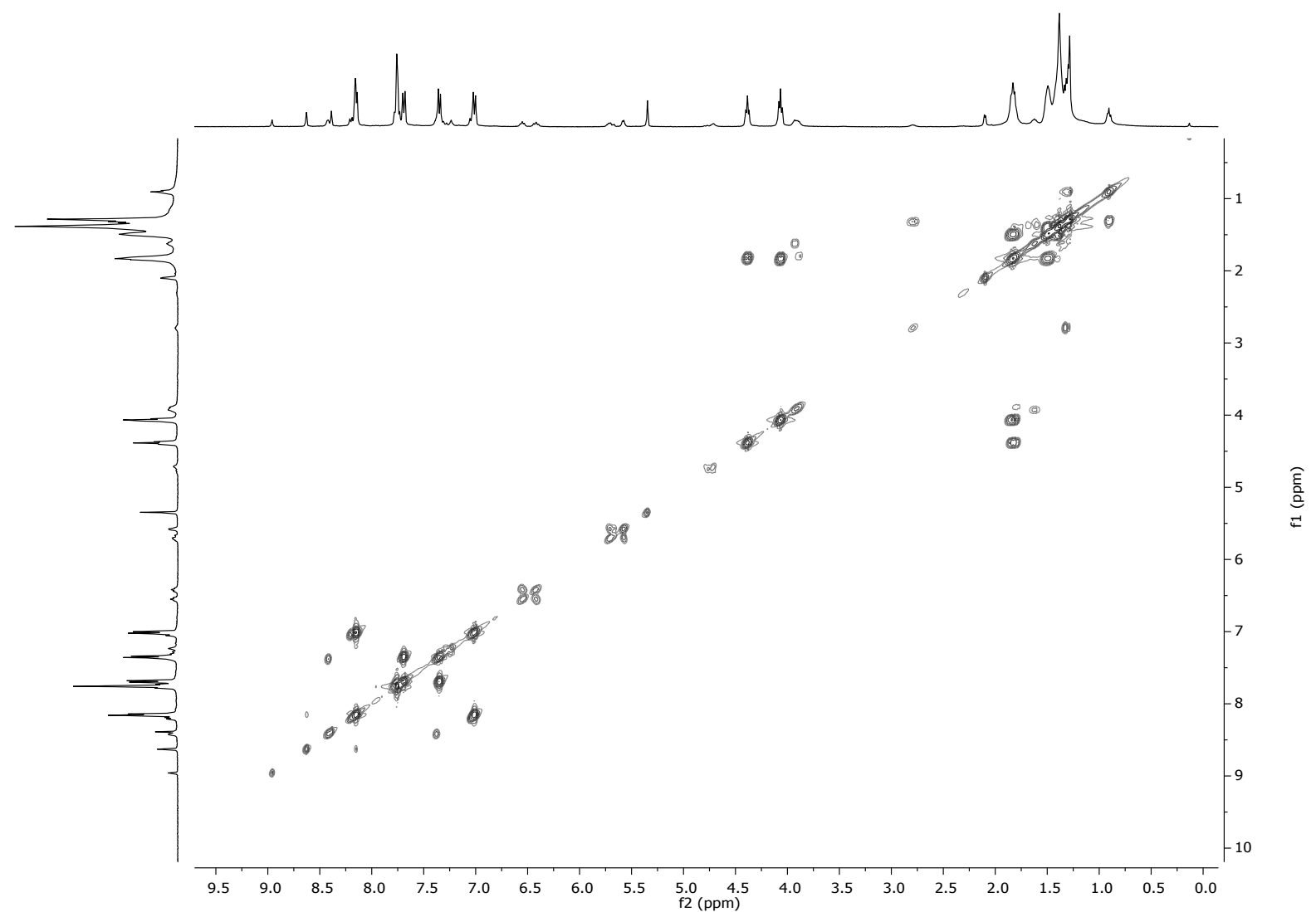

Figure S36. 2D COSY spectrum of $\mathbf{M}_{\mathbf{2}} \mathbf{L}_{\mathbf{2}}\left(400 \mathrm{MHz}, \mathrm{CD}_{2} \mathrm{Cl}_{2}, 298 \mathrm{~K}\right)$.

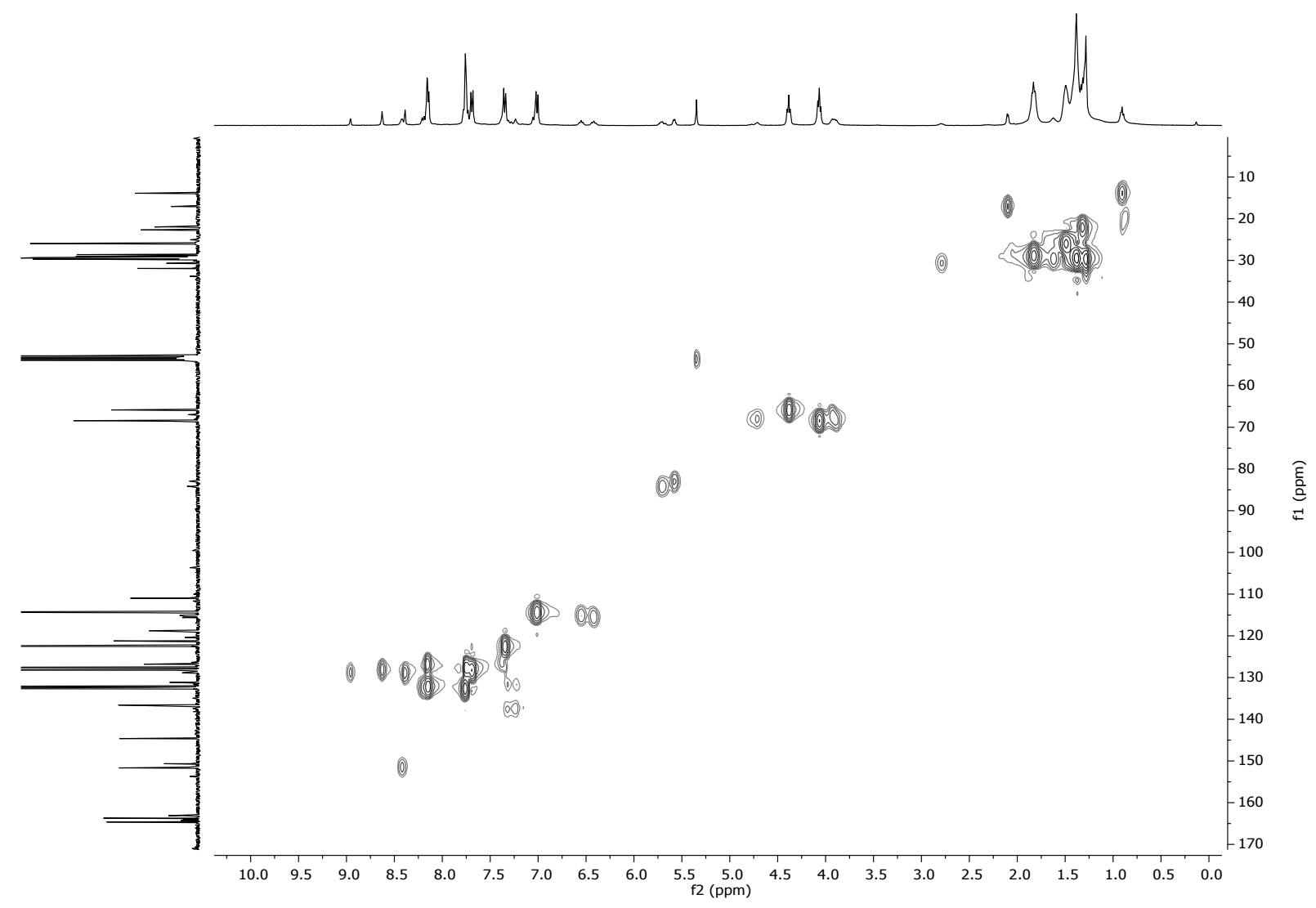

Figure S37. 2D HSQC spectrum of $\mathbf{M}_{\mathbf{2}} \mathbf{L}_{\mathbf{2}}\left(100 \mathrm{MHz}, \mathrm{CD}_{2} \mathrm{Cl}_{2}, 298 \mathrm{~K}\right)$. 


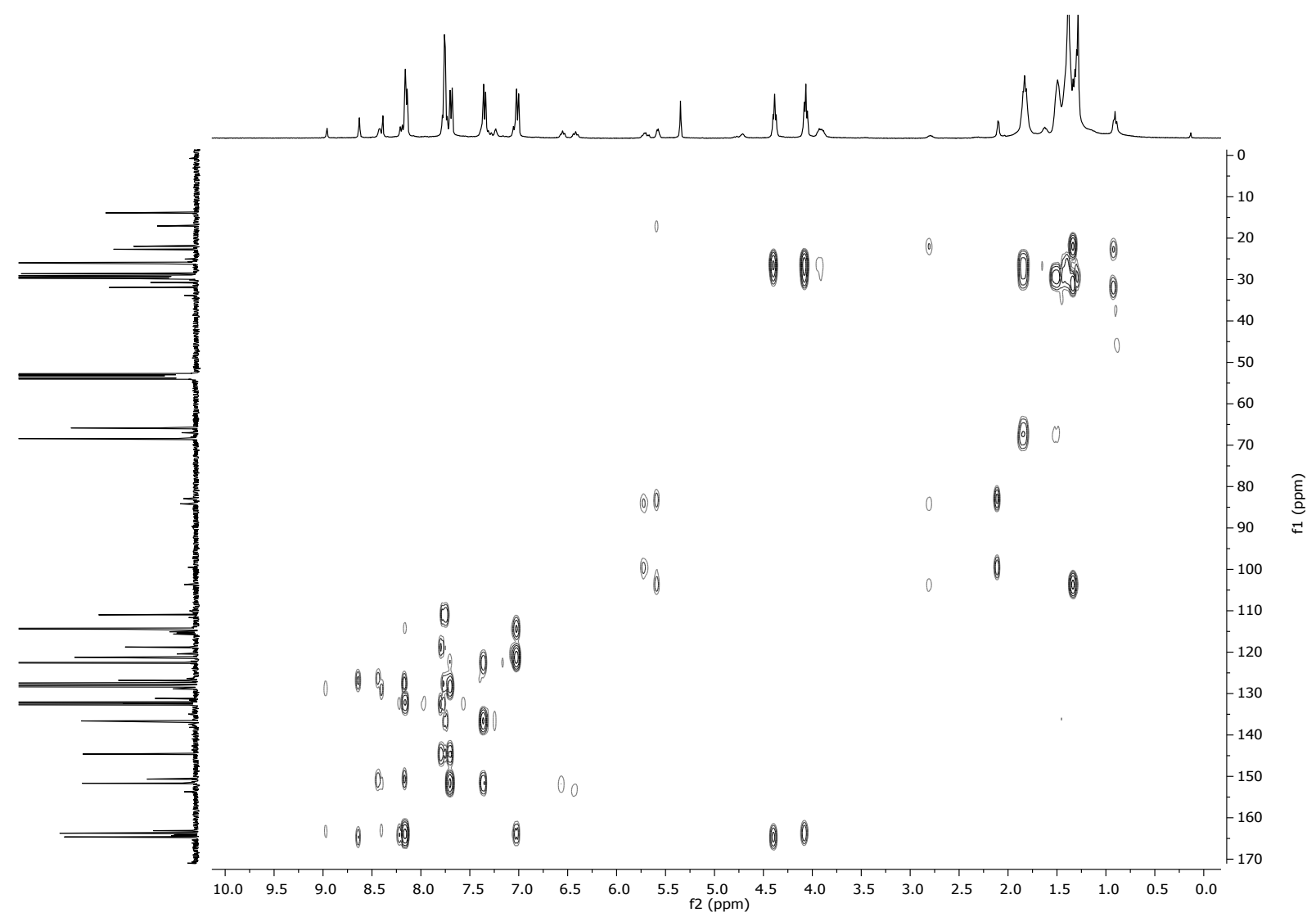

Figure S38. 2D HMBC spectrum of $\mathbf{M}_{\mathbf{2}} \mathrm{L}_{\mathbf{2}}\left(100 \mathrm{MHz}, \mathrm{CD}_{2} \mathrm{Cl}_{2}, 298 \mathrm{~K}\right)$. 


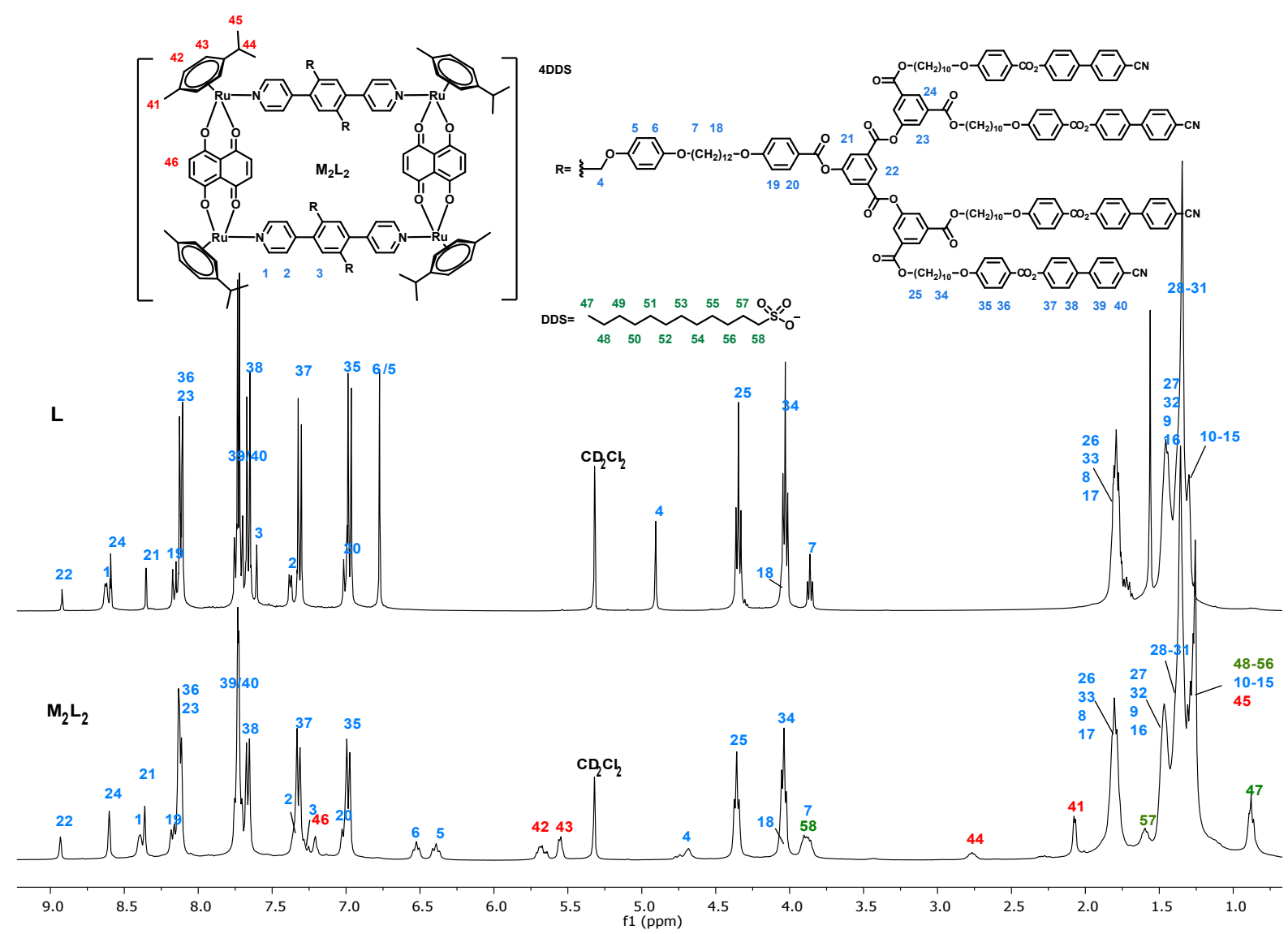

Figure S39. ${ }^{1} \mathrm{H}$ NMR spectra (400 MHz, $\mathrm{CD}_{2} \mathrm{Cl}_{2}, 298 \mathrm{~K}$ ) of $\mathbf{L}$ (top) and the metallacycle $\mathbf{M}_{\mathbf{2}} \mathbf{L}_{\mathbf{2}}$ (bottom); for simplicity, the numbering scheme is the same for $\mathbf{L}$ and for $\mathbf{L}$ in $\mathbf{M}_{\mathbf{2}} \mathbf{L}_{\mathbf{2}}$. 


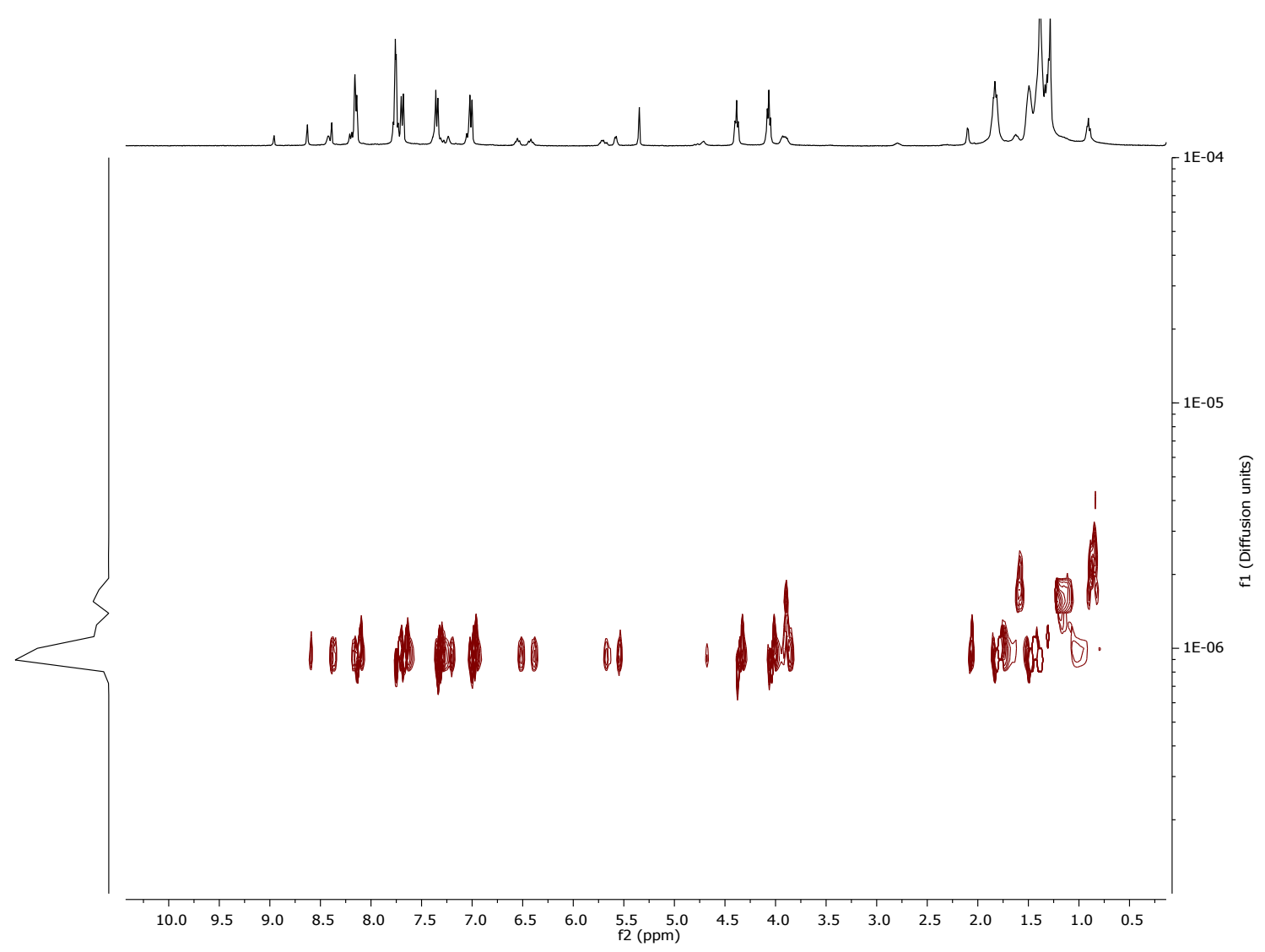

Figure S40. ${ }^{1} \mathrm{H}$ DOSY spectra (400 MHz, $\mathrm{CD}_{2} \mathrm{Cl}_{2}, 298 \mathrm{~K}$ ) of $\mathbf{M}_{\mathbf{2}} \mathbf{L}_{\mathbf{2}}$.

${ }^{1}$ Otsubo, T.; Kohda, T.; Misumi, S. Bull. Chem. Soc. Jpn. 1980, 53, 512.

${ }^{2}$ Xu, J.; Toh, C. L.; Liu, X.; Wang, S.; He, C.; Lu, X. Macromolecules 2005, 38, 1684.

${ }^{3}$ Dardel, B.; Guillon, D.; Heinrich, B.; Deschenaux, R. J. Mater. Chem. 2001, 11, 2814.

${ }^{4}$ Moore, J. S.; Stupp, S. I. Macromolecules 1990, $23,65$.

${ }^{5}$ Barry, N. P. E.; Therrien, B. Eur. J. Inorg. Chem. 2009, 4695.

${ }^{6}$ (a) Pieper, P.; Russo, V.; Heinrich, B.; Donnio, B.; Deschenaux, R. J. Org. Chem. 2018, 83, 3208. (b) Russo, V.; Pieper, P.; Heinrich, B.; Donnio, B.; Deschenaux, R. Chem. Eur. J. 2016, 22, 17366.

7 Han, Y.-F.; Jia, W.-G.; Lin, Y.-J.; Jin, G.-X. Organometallics, 2008, 27, 5002. 